\title{
Flexibility and Real Options Analysis in Emergency Medical Services Systems Using Decision Rules and Multi-Stage Stochastic Programming
}

\begin{abstract}
A novel approach to EMS infrastructure systems design, planning, and operations under long-term uncertainty is introduced based on multi-stage stochastic programming and decision rules, accounting for strategic flexibility (also known as real options - RO). Different from standard RO analysis, the approach mimics real-world decision-making by exercising flexibility based on conditional-go decision rules. The objective is to minimize the expected total costs over the system's life cycle, and the outputs are the optimal initial configuration and decision rules. A flexible solution provides lower expected cost than stochastically optimal rigid solutions, especially valuable when required incident coverage rate is $>95 \%$.
\end{abstract}

Keywords: flexibility in engineering design, real options analysis, Emergency Medical Service, multistage stochastic programming, decision rules.

\section{Introduction}

As part of public services, Emergency Medical Services (EMS) systems are significant for the wellbeing of cities and societies. EMS systems aim to provide quick and efficient out-of-hospital medical care to emergency patients and transport them to the nearest hospital if needed. Ong et al. (2009) illustrated that delivering fast defibrillation in medical emergencies could significantly increase patients' survival rate. Managing such EMS systems, however, can be challenging in both the short and long term. Significant uncertainty in the short term - such as incident patterns, geographical locations, and severity of incidents - influences the daily operations of the system (e.g., how to assign an emergency vehicle to respond to an incident). At the same time, EMS systems may also suffer from longer-term uncertainty related to changes in demographics, which influences the overall need for emergency services over time (e.g., where to allocate emergency vehicles and stations over time and space), and the shorter term uncertainty patterns as well. Indeed, an EMS system designed and deployed in view of short-term uncertainty patterns may prove sub-optimal in the long term. This reality is especially true in many cities of emerging countries, such as China and India, which need to deploy EMS systems on a large scale in the future to accommodate the growing needs of their populations. Hung et al. (2009) discussed EMS systems in China and suggested a few directions (e.g., training of EMS staff) to help EMS systems adapt better to growing urbanization. The study also indicated that smaller cities and rural areas were unable to meet Chinese regulatory standards due to the costs of construction and equipment acquisition, suggesting the need for more cost-effective 
designs for EMS systems. Sharma and Brandler (2014) reviewed current EMS systems in India and highlighted weak points requiring potential improvements (e.g., financial/budget limitations). Although it is the second most populous country in the world, there is currently no guideline for the training and operation of EMS systems in India, and the EMS system in Rajasthan received a total of 28,262 complaints in 2013 due to its low efficiency (Sharma and Brandler, 2014).

EMS system designs that planned to accommodate short-term variations in incident patterns (e.g. such as the daily fluctuations in incident rates) may not account well for the variations that occur over a longer time horizon (i.e. rising incident rates over several quarters and years). Hence, while performing well in the short term, such systems may perform in a sub-optimal manner in the long run due to a phenomenon referred as the "Flaw of Averages" (Savage, 2002). Indeed, EMS designs that rely heavily on the "average" or "most likely" scenario forecast for capacity planning and operations may perform well for some time when the future does not depart significantly from expectations. They may start performing badly if the future does not arise as planned, either having too much and unused capacity, or being under capacity, depending on how incident patterns evolve over time.

To deal pro-actively with this issue, one approach is to enable the system's capacity and configuration to change to future possibilities. This method is inspired from the concept of flexibility in systems design, or real options, which provides the "right, but not the obligation, to change the system easily in the face of uncertainty" (Trigeorgis, 1996). Not only does flexibility enable a system to capitalize on upside opportunities, it also helps limit potential exposure and risks to downside conditions, with the net effect of improving its overall expected performance. For example, modularity can be exploited whereby EMS stations - where emergency vehicles are operated and maintained - are initially deployed with smaller size and operational/maintenance capacity (e.g. 2 vehicles), and then expanded as needed, depending on how generation patterns evolve in the area serviced by the station. Such modularity requires careful planning for future upgrades, which is where infrastructure design and management is important. It allows the size of the station to be upgraded more easily, only if and when needed. This expansion reduces the initial capital cost, but also provides better contingencies to deploy additional capacity over time and space in a given area (i.e. city or region) to maintain or improve response time if the number of requests increases (i.e., upsides). This further deployment could be postponed if the number of incidents remains at the same level, reducing possible unused capacity and costs (i.e., downsides).

Although flexibility in engineering design sounds like a promising approach to deal with uncertainty, exercising flexibility strategies appropriately (e.g., gradually deploying the capacity of a station at the right times) can be challenging. This is because standard real options analysis (ROA) tools based on dynamic programming (DP) that are typically used to analyze flexibility and determine optimal timings for exercise, as well as design configurations, may not be fully suited for this purpose. For 
example, standard ROA tools typically rely on the assumption of path independence, which is not suitable to an engineering context (Wang and de Neufville, 2005) - see Section 2.3 for more details. Also, in practice, one may need to be proficient at using DP to implement the optimal policies obtained from an optimization analysis. When dealing with multiple uncertainty sources and flexibility strategies, applying standard ROA tools like multinomial lattices and approximate dynamic programming can be challenging due to the curse of dimensionality. For these reasons, standard ROA tools may prove difficult to use to analyze flexibility in EMS systems, which typically involve considerations of multiple sites (i.e. stations), vehicles, and flexibility (or real option) strategies. Thus, the potential of flexibility to generate better performances may not be fully recognized and exploited under standard ROA methods.

To alleviate concerns related to the ability to quantify the value of flexibility, finding the optimal flexible system configuration and times to exercise flexibility, a novel approach based on decision rules was recently proposed by (Cardin et al., 2017a). The authors developed a multi-stage stochastic programming model to capture the decision-making dynamics, while flexibility strategies were exercised based on conditional-go decision rules. This approach is practical as it provides planners with straightforward and intuitive guidelines for operations. For example, it helps determine the stochastically optimal parameter to determine the right timing and amount to deploy additional capacity based on a conditional-go decision that is similar to an IF-THEN-ELSE statement in computer programming (e.g. IF incident rates reach threshold $\mathrm{X}, T H E N$ upgrade station capacity to $\mathrm{Y}$, ELSE do nothing). EMS systems are more complex in terms of the number of decision variables and interactions that are considered in the study by (Cardin et al., 2017a), and typically involve deploying infrastructure and emergency vehicles over multiple sites to accommodate needs for emergency services. This provides an opportunity for further methodological improvement and application.

Considering the background above, the main contribution of this paper is to propose a novel approach based on multi-stage stochastic programming to systematically analyze real options and flexibility in EMS systems, considering long-term uncertainty in incident pattern generation. Such a time horizon is typically longer, in the order of several quarters to years for planning purposes. The design problem considers where and when to install/open new vehicle stations (strategic), where to deploy emergency vehicles (tactical), and whether to purchase new vehicles (strategic) subject to how incident patterns evolve over time. This approach also exploits a decision rule technique, with considerations of the fact that large-scale urban systems will typically require capacity deployment and operations over multiple sites and time periods. Various flexibility strategies associated with typical decision-making, such as phasing/staging deployment and capacity expansion, are taken into account and exercised using decision rules. As a demonstration of this approach, a case study is conducted in the context of a hypothetical city in an emerging country, using data for the input parameters (e.g., locations of 
candidate sites) from a published study, and publicly available information on incident data in major cities.

This paper is organized as follows. Section 2 presents a literature review of the relevant work in optimal capacity planning and operations in EMS systems, standard ROA tools, as well as flexibility in engineering design. Section 3 revisits standard ROA techniques and extends the tools to capacity planning problems at multiple sites that is better suited to analyze flexibility in EMS systems. The novel framework proposed in this paper is introduced in Section 4, along with related assumptions and a generic form for the mathematical model. Numerical experiments are discussed in Section 5, and the presentation consists of a description of the optimal solution, performance, and computational times required. A comparison between the results obtained from standard ROA tools and the proposed approach is provided, in addition to a sensitivity analysis on important modeling parameters. Section 6 then presents the concluding remarks, limitations, and future directions for research.

\section{Related Work}

The literature review below consists of four sub-sections. The first three sub-sections review past research that is tightly connected to the approach used in this paper. Specifically, the first sub-section discusses relevant work related to EMS systems, from short-term to long-term uncertainty considerations. The second sub-section focuses on introducing the standard real options analysis approaches and the limitations on implementation and analysis of real-world EMS systems. The third sub-section opens up a discussion on the background related to flexibility in engineering design, including applications of this concept. Then, an overview of the main contributions is discussed.

\subsection{Emergency Medical Services (EMS) Systems}

As a significant part of urban systems, EMS systems play an important role in daily life and have been widely studied for decades. The literature is extensive (e.g., Başar et al. (2012); Farahani et al. (2012); Li et al. (2011)), thus the discussion in this paper is limited to work related to optimal capacity planning and operations (also known as resource allocation) in EMS, where operations research (OR) methods are most widely applied. Allocation problems mainly fall into two categories: minimizing resources subject to service requirements and maximizing system performances under limited resources. Toregas et al. (1971) introduced a deterministic set covering location problem (SCLP) to minimize the number of facilities, while Church and ReVelle (1974) proposed a maximal covering location problem (MCLP) to maximize the population covered by the emergency service. Daskin (1983) extended the work of Church and ReVelle to the maximal expected coverage location problem (MEXCLP) by introducing the fact that an ambulance can be busy with a certain probability. Ball and Lin (1993) assessed the service level of each individual demand point by a chance constraint under the assumption of a new better than used distribution. To capture the random nature of emergency 
requests (i.e., demand), queuing theory, probabilistic constraints, stochastic programming, and robust optimization have been applied in various studies (An et al., 2015; Baron et al., 2009; Beraldi and Bruni, 2009; Chen and Yu, 2016; Iannoni et al., 2015; Iannoni and Morabito, 2007; Liu et al., 2016; Shishebori and Yousefi Babadi, 2015). Some studies considered other important features of the EMS system, such as multiple types of emergency vehicles or uncertain travel time, to make the analysis more realistic (Berman et al., 2013; McLay, 2009). Nair and Miller-Hooks (2009) conducted a realworld analysis using data from Montreal, Canada, and quantified the benefits of considering relocating emergency vehicles between calls over the course of a day due to the demand patterns and changes in travel time.

There is much less research, however, accounting for capacity deployment over time with considerations of long-term uncertainty than there is accounting for capacity deployment in the short term. Gunawardane (1982) extended SCLP and MCLP to dynamic versions under the assumption of deterministic emergency requests, while Başar et al. (2011) developed a dynamic version of MCLP to manage an EMS system in Istanbul, Turkey. In both studies, it was assumed that a facility would not be closed once it was opened after the first period, and that a facility existing since the first period would not be reopened if it was closed. Farahani et al. (2009) discussed a multi-reallocation problem with regards to a single facility in discrete time horizons where emergency requests were weighted according to a function of traveling time. Ghaderi and Jabalameli (2013) proposed a budgetconstrained model to minimize the travel and operational costs over the life cycle of an EMS system.

Besides EMS systems, location and relocation models are also important for other emergency services. Chow and Regan (2011c) proposed a $k$-server $p$-mean dynamic relocation model to tackle a wildland fire-planning problem. Different from typical MCLP, their model captured the exact decision making based on a more detailed requirement, and also introduced a chance constraint to guarantee the minimal probability of coverage on demand. Little research has accounted for flexibility in a dynamic capacity and resource allocation process, except for (Cardin et al., 2015a), in which an EMS system was analyzed by a simulation game rather than OR techniques.

\subsection{Standard Real Options Analysis (ROA) Approaches}

The valuation of flexibility is significant for applying this concept to a design since it normally requires a trade-off between the cost premium for enabling flexibility and the benefits. Standard Discounted Cash Flow (DCF) analysis does not account for inevitable changes and flexibility strategies since typically deterministic, and it thus is not well suited for valuing flexibility (Trigeorgis, 1996). To fill this gap, ROA was developed from the theory of financial options to quantify the value of flexibility, such as the Black-Scholes (B-S) model and other tools based on dynamic programming (Copeland and Antikarov, 2001; Dixit and Pindyck, 1994; Myers, 1984). 
Standard ROA such as decision analysis and lattice-based approaches are based on dynamic programming, rely on a folding back process to calculate the best design decision using Bellman's equation (Bellman, 1952). Figure 1 illustrates a three-period binomial lattice model with consideration of one uncertainty. $S$ denotes the initial state of uncertainty, which could be either monetary (e.g., costs, prices) or non-monetary (e.g., emergency requests, electricity demand). Parameters $\mu$ and $d$ represent the up and down factors scaling the response from the previous period, with corresponding probability $p$ of going up, and probability $1-p$ of going down, where $0<p<1$. This lattice-based approach has been widely used in various sectors; see, for example, Acciaro (2014); Khansa and Liginlal (2009); Rau and Spinler (2016).

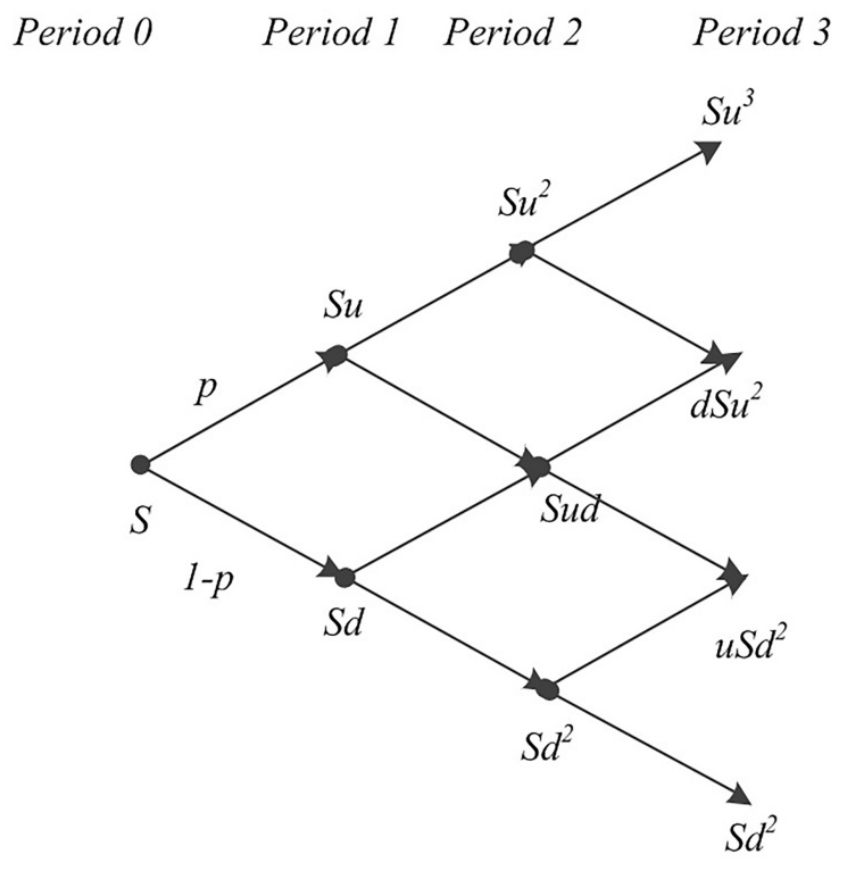

Figure 1. An illustration of a three-period binomial lattice model.

In addition to standard ROA, simulation and approximate approaches are also used to analyze the value of flexibility. de Neufville et al. (2006) developed a spreadsheet based Monte-Carlo simulation to evaluate the capacity expansion flexibility for a parking garage case study. Chow and Regan (2011b) proposed a real option model incorporating a deferral option to address managerial flexibility in transportation planning, and demonstrated the model on the classic Sioux Falls, South Dakota, network. Moreover, Chow and Regan (2011a) incorporated real option methodologies into network modeling, proposed two models, and solved them using least squares Monte-Carlo simulation. One model considers deferral options on investment, while the other considers a set of deferral options that link components of a network design as individual options. Chow and Sayarshad (2016) proposed two reference policies regarding non-myopic sequential network and applied approximate dynamic programming to tackle the sampling issue. Powell et al. (2012) presented a modeling framework 
based on sequential stochastic optimization that potentially positioned most algorithmic strategies into four fundamental classes of policies. The framework was then applied to the problems in the context of transportation and logistics, indicating that different policies work well under the same conditions. Different from our proposed approach, these papers focus on general transportation planning problems and example real options "on" projects (i.e. investment deferral), which are flexibilities existing from an overall managerial standpoint (Wang and de Neufville, 2005). In contrast, real options "in" projects (which are the focus of this paper) require careful design and planning to be exercised in operations (e.g. buying additional land or preparing for shared infrastructures in preparation for a possible flexible capacity expansion in existing EMS stations).

Standard ROA as described above is not well suited to analyze flexibility in EMS systems. For one, it assumes path independence (see Figure 1) to reduce the curse of dimensionality. In doing so, it moves away from the reality that engineering systems typically behave in a path dependent manner. That is, an up-down movement of the main stochastic variable as shown in Figure 1 may not lead to the same value and system performance as a down-up movement, as assumed in standard ROA. In the binomial model, it is assumed that the value of a node at period $t$ with an up-down movement in the previous two periods $(t-2 \Delta t, t-\Delta t)$ is equivalent to that of a node with a down-up movement. Although this is true in the context of financial options, this assumption does not hold true in an applied context because system operators and planners may act differently in these two scenarios. For instance, one may expand system capacity to meet growing emergency calls, in which case the system would have extra capacity when the number of calls drops subsequently. This leads to a different performance if a down-up movement had occurred, and no additional station capacity was added. In essence, an updown sequence may lead to the creation of new artefacts (e.g. additional stations and vehicles), while a down-up sequence may lead to no more capacity being introduced, leading to very different performance outcomes in both cases. Another issue with standard ROA techniques is that there is no complete and efficient market to trade the physical assets, which provides grounds for the arbitrageenforced and risk neutrality assumptions that are crucial to the underlying economic theory.

Another challenge in using standard ROA to analyze EMS systems lies in the form of the solution when multiple uncertainty sources and flexibility strategies are considered. As described in Section 3, the optimization analysis requires the development of a multinomial lattice. A backward induction process across the different lattices must be performed to determine the optimal decision at each point in time, which may be difficult to execute for decision-makers not acquainted with the techniques. Given that multiple lattices must be considered at the same time also increases the potential for confusion in determining the optimal course of action. In contrast, the decision rule technique introduced in this paper only requires decision-makers to follow the state of a particular random variable (e.g. incident rates) and react dynamically, using the recommended and stochastically optimal 
design and decision rule variable parameters - see additional explanations on decision rule ROA in Section 2.4 .

\subsection{Flexibility in Engineering Design}

Flexibility in engineering design (also referred to as real option) is a promising approach to proactively deal with uncertainty in EMS systems by enabling the system to change and adapt in the face of uncertainty (Saleh et al., 2009). Inspired conceptually from standard ROA theory, it aims to embed flexibility in the early design and planning phases of engineering systems so as to improve their expected lifecycle performance. Cardin (2014) proposed a five-phase design framework and taxonomy for systematically enabling flexibility in complex systems, along with a literature review of past related works. As mentioned above, real options can typically be categorized into two groups: real options "in" projects and real options "on" projects. The former focuses on changing the physical design of systems to deal with uncertainty, such as staging/phasing deployment of capacity and upgrading/reducing capacity over time and space. This type of flexibility thus requires an in-depth understanding of the system itself. In contrast, the latter group focuses on managerial level decisionmaking, like deferring investment until favorable market conditions arise, which treats the system as a black box (Wang and de Neufville, 2005).

Using a capacity expansion flexibility strategy as an example, the benefits of applying flexibility play on two sides of uncertainty. One the one hand, the flexibility enables planners to gradually upgrade the EMS system to meet growing incident rates in different sectors, due to demographic and other changes, so as to improve the expected response time, and minimize initial infrastructure costs. Indeed, too few stations and emergency vehicle capacity may lead to longer response times, on average, if such rates are increasing. On the other hand, flexibility limits risks and reduces the potential of unused capacity, in case incident rates grow slower than anticipated. This also reduces the risks of higher capital costs arising from such unused capacity.

Playing in such a way on both sides of uncertainty has the net effect of improving the expected lifecycle performance of the system, routinely by $10-30 \%$ as compared to the solution obtained without considerations of flexibility, be it measured in terms of economics (e.g. cost savings or profits), or others (e.g. response time). Similar observations have been made in many studies and industry sectors, including the automotive, real estate, energy, oil and gas industries (Cardin and $\mathrm{Hu}$, 2016; Cardin et al., 2015b; de Neufville and Scholtes, 2011; Luo, 2015). In network research, Chow et al. (2011) proposed a real option portfolio management framework for an adaptive network planning problem. The framework was an extension of Smit's and Trigeorgis' option framework, incorporating network synergies, and it was applied to a real network problem in Iran. Only a few 
studies have focused on applying real options in the EMS sector (Cardin et al., 2015a), however, and none of them have analyzed the flexible capacity deployment problem in EMS systems in an analytical manner, as done in this study.

It should be noted that flexibility may not always be favorable due to the high cost premium for enabling flexibility, and the fact that the value of flexibility may also depend on how one sets up the "benchmark". Kort et al. (2010) found that flexibility to split a project into multiple steps and to choose the timing of each step individually was dominated by a one-step investment plan. This may occur, for instance, when economies of scale are particularly strong, or when the discount rate used to account for the time value of money is low, providing no incentive to defer the capacity deployment cost. As shown by the authors, the level of uncertainty may also affect the value of flexibility significantly. Our studies provide complementary views as they both analyze the combinations of factors that may affect the value of flexibility (positively or detrimentally), although our work focuses on the conditions of EMS systems as opposed to general capacity deployment problems - see Section 5 for more details.

\subsection{Main Contributions}

The main contribution of this paper is a novel modeling framework to support the evaluation of flexibility and real options in the context of multiple facilities allocation problems, with considerations of long-term uncertainty in an EMS design problem. More specifically, the framework builds upon and extends previous work on long-term EMS systems design (as an example of multifacility deployment problem) and analysis, as summarized in the literature review of Section 2.1, by considering and modeling uncertainty in the long term (i.e. yearly), through modeling of the incident rates based on historical data, which are then used to model incident patterns over a shorter time period (i.e. quarterly). Considering long-term uncertainty aims to help designing an EMS system that is stochastically optimal over its life cycle in terms of KPIs. As compared to the work done in standard ROA and flexibility analysis summarized in Sections 2.2 and 2.3, this work provides an additional layer of analysis beyond existing work (also related to the work on EMS system in Section 2.1) by modeling real options in the form of flexibility strategies, with particular focus on real options "in" EMS systems. These enable EMS planners and operators to recognize the value associated with the ability to adapt to changing conditions, and the careful planning from a design standpoint that is required to accommodate such strategies at the infrastructure level. To these authors' knowledge, this is the first study that provides such combined analysis and contributions for EMS systems design and operations operating under uncertainty, with explicit considerations of flexibility modeled as a real option. The framework captures decision-making via a multi-stage stochastic model, while flexibility is modeled and exercised in operations based on decision rules. This framework produces solutions readily usable to planners and system operators, in the form of "IF-THEN-ELSE" conditional-go 
decision rules, and demonstrably leads to similar valuation outcomes and solutions as standard ROA techniques, as shown below for relatively small-scale problems (see Table 2).

\section{Revisiting Standard ROA in Multi-Facility Capacity Deployment}

This section describes standard ROA approaches so the reader may gain a better perspective on the differences with our proposed approach. As introduced in Section 2.2, standard ROA tools include the B-S model and dynamic programming approaches in which lattice-based methods are widely used for the valuation of flexibility. The literature review showed that lattice-based approaches are rarely used to analyze the capacity deployment problem in a multi-facility context, mainly due to the curse of dimensionality. More specifically, the dimensions of the tree structures can quickly become intractable with an increase in the number of sites and uncertainty. Figure 2 illustrates two examples of structures for lattice-based approaches when dealing with a multi-facility capacity deployment problem. The approach in the upper Figure 2(a) analyzes the problem using a standard binomial lattice, where each lattice tree represents one facility or candidate site. $S$ denotes the initial state of the main uncertainty drivers (e.g. incident rate at a given location). This approach, however, cannot capture the possible interactions between different facilities or sites, as is the case for most EMS systems. Such systems must be planned and operated as a whole in a given city or district, so as to ensure best coverage at limited cost. The binomial approach here leads to a conservative and unrealistic result, as it treats facilities separately rather than as a coherent system.

The second approach is the so-called multinomial lattice, which puts all uncertainty drivers (and their corresponding sites) into one node. This approach is consistent with analyzing an EMS as an integrated system, rather than a collection of independent sites and vehicles. The number of branches of a node therefore depends on the number of uncertainties (or sites), and is usually greater than two (i.e., binomial). Figure 2(b) shows a capacity deployment problem associated with two facilities or uncertainties (i.e., $S_{1}$ and $S_{2}$ ), where the uncertainty at each facility is identical and independently distributed (iid). As can be seen, there are five possibilities for each node for the next period, in which four of them consider an up or down scenario for every uncertainty source. This approach accounts for interactions because the state of subsequent nodes depends on decision-making for the anterior node with considerations of all facilities together. As a lattice-based approach, the number of scenarios in a multinomial lattice is fairly large since it increases exponentially along with the number of uncertainties and planning periods. 

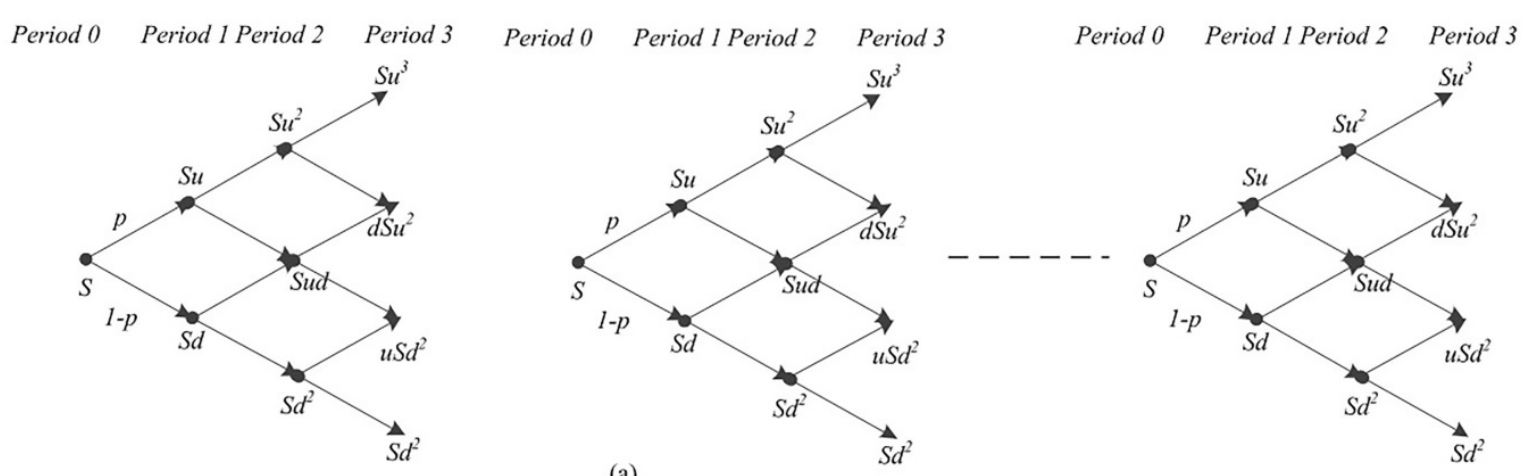

Period $0 \quad$ Period $1 \quad$ Period 2

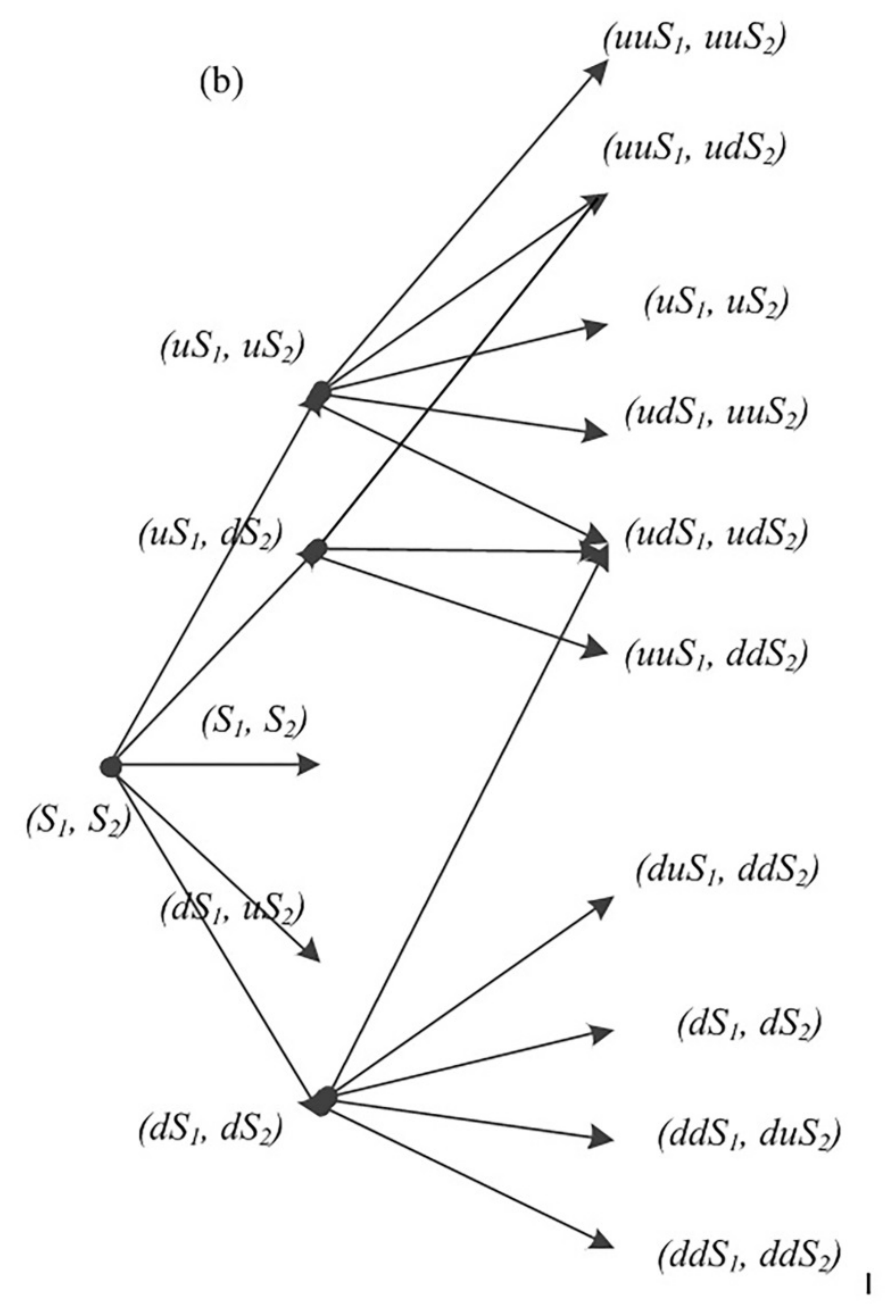

Figure 2. Examples of structures for lattice-based approaches to ROA. (a) The structure of the binomial lattice-based approach; (b) the structure of the multinomial lattice approach.

To tackle the curse of dimensionality and increased computational complexity, approximate dynamic programming (ADP) can be used. The original objective function is approximated by a related but simpler function to make it computationally feasible. As shown in Equation (1), the original optimal function $J_{t+1}$ is replaced by an approximation $\tilde{J}_{t+1}$ (i.e., value function approximation). The value 
function approximation is used to approximate the complex objective function at state $t$, which can take on various forms (Powell, 2009). Note that the value function should have the same property as the original objective function (e.g., convexity or concavity). This equation denotes the optimal solution starting from period $t$ until the last period, where $x_{t}$ and $w_{t}$ are the decision variable and random (stochastic) variable, respectively. $u_{t}$ represents the constraints associated with varaible $x_{t}$, while function $g($.$) is the cost function at period t$.

$$
\tilde{J}_{t}\left(f_{t-1}\left(x_{t-1}, u_{t-1}, w_{t-1}\right)\right)=\min _{u_{t} \in U_{t}\left(x_{t}\right)} E\left\{g_{t}\left(x_{t}, u_{t}, w_{t}\right)+\tilde{J}_{t+1}\left(f_{t}\left(x_{t}, u_{t}, w_{t}\right)\right)\right\}
$$

Theoretically, this approximation could be iteratively updated via simulation using statistical (either parametric or nonparametric) models (e.g., linear regression) based on past observations. (Cardin et al., 2017a) used a least-square policy iteration (LSPI) algorithm to analyze a design problem comprised of multiple uncertainties and flexibility strategies for a single infrastructure facility. This algorithm is a reinforcement learning algorithm designed to solve a practical control problem with relatively little training data (Lagoudakis and Parr, 2003). It is applied here in the numerical analysis as an alternative tool to standard ROA for solving the capacity deployment problem with consideration of multiple facilities and flexibility strategies.

\section{Modeling Framework Description}

\subsection{Proposed Framework}

This section introduces the proposed modeling framework in generic form for analyzing the value of flexibility in EMS systems based on decision rules. A main assumption developed here is inherited from multi-stage programming: decision-making responses based on past observations. Any information not known at time $t$ is not considered and thus will not affect the decision making process. As the most important benefit of the framework, flexibility in engineering design enables managers to deal pro-actively with uncertainty by changing the system in the face of uncertainty. Many flexibility strategies can be incorporated and analyzed, such as expansion options, deferral options, and abandonment options. The framework also enables simultaneous evaluation of multiple flexibility strategies subject to different uncertainty sources in a way that would be otherwise difficult to do using standard methods based on multinomial lattice analysis. 


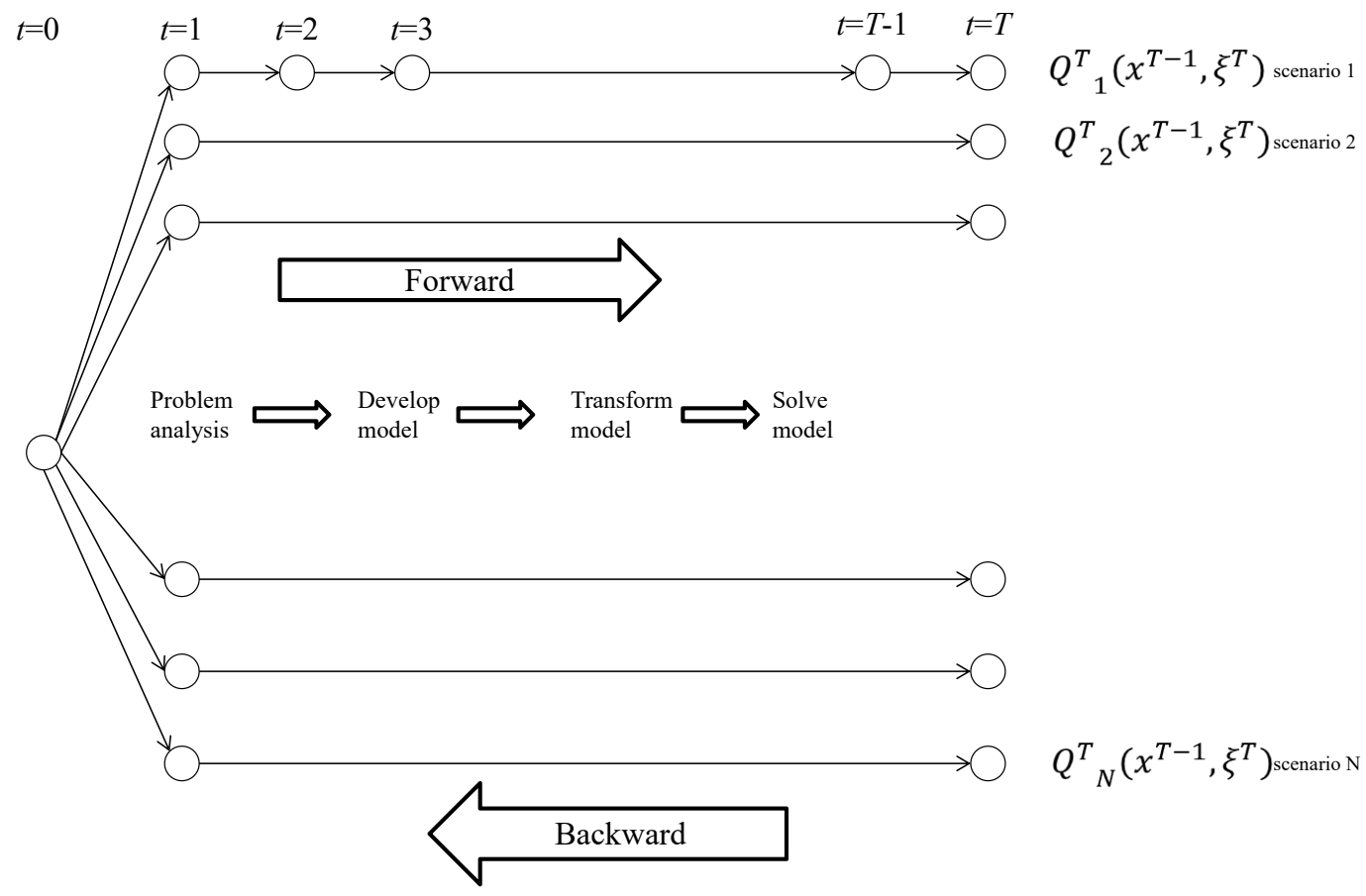

Figure 3. Flowchart of the proposed framework to analyze EMS systems under uncertainty and flexibility

Figure 3 above illustrates the flow of the framework. The first step is to identify the problem based on domain expertise and/or external discussions with experienced EMS providers. After having an understanding of the design problem, the next step is to develop a multi-stage stochastic programming model. The formulation will be introduced later in this section. The original mathematical model may not be straightforward and could be difficult to solve. In that case, one possible approach is to transform the original model into an equivalent simplified form, or using an approximation, which highly depends on the complexity of the design problem and the structure of the modeling. As can be seen in Figure 3, the original model here can be transformed into multiple independent sub-problems, based on the concept of sample average approximation. Instead of solving the entire problem once, the proposed approach helps solve sub-problems in parallel in a more efficient manner. Note that the formulation is forward looking, but the solving may be following a backward induction process, as discussed later.

Let the stochastic process $\xi^{t} \in \mathbb{R}, t=1, \ldots, T$, denote the uncertain information (e.g., emergency incidents), and $x^{t}$ be the vector of decision variables chosen at period $t$ based on the information up to now (i.e., $\xi^{[t]}$ ). A typical decision process with a planning horizon of $T$ periods can be described as: observation $\left(\xi^{[1]}\right) \rightsquigarrow$ decision $\left(x^{1}\right) \rightsquigarrow$ observation $\left(\xi^{[2]}\right) \rightsquigarrow$ decision $\left(x^{2}\right) \cdots \cdots$ observation $\left(\xi^{[T]}\right) w$ 
decision $\left(x^{T}\right)$. Decisions at period $t$ only rely on past observations rather than future forecasts, which makes the process more realistic. The objective of this capacity deployment problem could then be naturally described by a generic minimization $T$-stage stochastic programming model (Ruszczyński and Shapiro, 2003)

$$
\min _{x^{1} \in \chi^{1}} f^{1}\left(x^{1}\right)+E\left[\inf _{x^{2} \in \chi^{2}\left(x^{1}, \xi^{2}\right)} f^{2}\left(x^{2}, \xi^{2}\right)+E\left[\ldots+E\left[\inf _{x^{T} \in \chi^{T}\left(x^{T-1}, \xi^{T}\right)} f^{T}\left(x^{T}, \xi^{T}\right)\right]\right]\right],
$$

driven by the set of uncertainty scenarios $\Xi$. Functions $f^{t}($.$) are continuous functions of interest to$ managers and planners (e.g., operating and capital costs), while functions $\chi^{t}, t=2, \ldots, T$, are measurable closed valued functions (i.e., real constraints). Equation (2) is well formed, yet it is intractable due to its complexity when $T$ is large (e.g., $T \geq 10$ ). This equation could also be written in a concise form of dynamic programming that looks at the problem backward from the last stage (Ruszczyński and Shapiro, 2003). In this study, it is further assumed that $\Xi$ is a finite set of $\left(\xi_{1}, \xi_{2}, \ldots, \xi_{S}\right)$ with a corresponding probability $\sum_{S=1}^{S} p_{s}=1, p_{s} \geq 0$, where $\xi_{S}$ is a sample scenario of uncertainty realization. Let $Q^{T}\left(x^{T-1}, \xi^{[T]}\right)$ denote the optimal solution of the last stage problem

$$
Q^{T}\left(x^{T-1}, \xi^{T}\right)=\min _{x^{T} \in \chi^{T}\left(x^{T-1}, \xi^{T}\right)} f^{T}\left(x^{T}, \xi^{T}\right) .
$$

Here $Q^{T}$ is the objective function of the last stage problem, which will be used in the previous stage for solving a similar optimization problem. Therefore, the previous stage of the last stage problem (i.e., stage $T-1$ ) can be formulated as

$$
\begin{aligned}
& \min _{x^{T-1}} f^{T-1}\left(x^{T-1}, \xi^{T-1}\right)+\sum_{s=1}^{S} p_{s} Q_{s}^{T}\left(x^{T-1}, \xi_{s}^{[T]}\right) \\
& \text { s.t. } \quad x^{T-1} \in \chi^{T-1}\left(x^{T-2}, \xi^{[T-1]}\right)
\end{aligned}
$$

based on the sample average approximation (SAA) assumption (Shapiro, 2013). Any $t$-stage subproblem of the original $T$-stage problem can thus be formulated in this way, and the first stage problem is as follows.

$$
\begin{gathered}
\min _{x^{1} \in \chi^{1}} f^{1}\left(x^{1}\right)+\sum_{s=1}^{S} p_{s} Q_{s}^{2}\left(x^{1}, \xi_{s}^{[2]}\right) \\
\text { s.t. } Q^{t}\left(x^{t-1}, \xi^{[t]}\right)=\inf _{x^{t} \in \chi^{t}\left(x^{t-1}, \xi^{t}\right)}\left[f^{t}\left(x^{t}, \xi^{t}\right)+\sum_{s=1}^{S} p_{s} Q_{s}^{t+1}\left(x^{t}, \xi_{s}^{[t+1]}\right)\right], t=2, \ldots, T ; \\
x_{k}^{1}=x_{l}^{1}, k \neq l, k, l \in S ;
\end{gathered}
$$




$$
x_{k}^{t}=x_{l}^{t}, k \neq l, \xi_{k}^{[t]}=\xi_{l}^{[t]}, k, l \in S, t=2, \ldots, T
$$

There will be a total of $S^{T-1}$ scenarios in Problem, and each antecedent node has $S$ subsequent nodes (scenarios). The non-anticipative constraints (e.g., $x_{k}^{1}=x_{l}^{1}$ ) hold if and only if the observations up to period $t$ are exactly the same. In the proposed modeling framework, the decision rules perform the same role as non-anticipative constraints. This is because the conditional-go decision rule will be exercised if the explicit requirements are triggered, which depends on the observations so far. Problem (5) is therefore the generic form of the modeling framework for capacity deployment problems. Note that the multi-facility feature is embedded in the decision vector $x^{t}=\left(x_{1}^{t}, x_{2}^{t}, \ldots x_{j}^{t}, \ldots, x_{J}^{t}\right)$, where $x_{j}^{t}$ is the vector of decision variables associated with site or facility $j \in J$.

For better understanding, consider a strategic planning horizon of $T$ periods over which requests for emergency services are random. The purpose of the problem is as simple as deploying emergency resources (e.g., ambulances, emergency stations) over the system's life cycle incorporating the concept of flexibility. Index $i$ denotes the district or emergency request node, and $j$ denotes the candidate site for installing an EMS station. The small superscript $t$ is represents a specific time unit (e.g., $t=1,2,3, \ldots$ ), while the capital superscript $T$ represents the set of time units (e.g., $|T|=20$ ). Let $\theta_{j s}^{t}$ denote the capacity deployed at site $j$ at period $t$ under scenario $s$, while $y_{i j s}^{t}$ denotes the assignment variables for which emergency requests occurring in district $i$ will only be responded to by emergency vehicles allocated at site $j$ at period $t$ under scenario $s$. The decision variable vector $x^{t}$ consists of $\theta_{s}^{t}$ and $y_{s}^{t}$ over all indices $i$ and $j$. The formulation of the last stage problem can be found as Problem (9) where continuous function $f^{t}($.$) is the linear combination of several costs functions$ $C^{t}(),. H^{t}($.$) , and M^{t}($.$) . Specifically, cost function C^{t}\left(\theta^{t}, \xi^{t}\right)$ presents the basic cost associated with the installation, operation, and maintenance of emergency stations and vehicles. Cost function $H^{t}\left(\theta^{t-1}, \theta^{t}\right)$ calculates the cost associated with flexibility strategies such as phasing deployment and capacity expansion. $M^{t}\left(y^{t}, \omega^{t}\right)$ is responsible for the the assignment cost for responding to emergency requests.

$$
\begin{gathered}
\min _{\theta_{s}^{T}, y_{s}^{T}, \omega_{s}^{T}} \frac{1}{(1+\lambda)^{T}}\left[C_{s}^{T}\left(\theta_{s}^{T}, \xi_{s}^{T}\right)+H_{s}^{T}\left(\theta_{s}^{T-1}, \theta_{s}^{T}\right)+M_{s}^{T}\left(y_{s}^{T}, \omega_{s}^{T}\right)\right] \\
\text { s.t. } \quad F_{s}^{T}\left(\theta_{s}^{T-1}, \xi_{s}^{[T]}, y_{s}^{T-1}\right)=\theta_{s}^{T}, \forall s \in S ; \\
G_{s}^{T}\left(\omega_{s}^{T-1}, \theta_{s}^{T}\right)=\omega_{s}^{T}, \forall s \in S ;
\end{gathered}
$$




$$
\begin{gathered}
L_{s}^{T}\left(\omega_{s}^{T}, \xi_{s}^{[T]}\right)=y_{s}^{T}, \quad \forall s \in S ; \\
H_{s}^{T}\left(\theta_{s}^{T-1}, \theta_{s}^{T}\right)=\left\{\begin{array}{ll}
K\left(\theta_{s}^{T}-\theta_{s}^{T-1}\right)^{\alpha}, & \text { if } \theta_{s}^{T} \geq \theta_{s}^{T-1} \\
0, & \text { otherwise }
\end{array}, \forall s \in S ;\right.
\end{gathered}
$$

$\lambda$ is the discount factor accounting for the time value of money, while functions $F, G$, and $L$ are the abstract form of constraints involved in the decision-making. The regular boundaries for an emergency request assignment are captured thoroughly in Function $L$ and $G$. For example, a request cannot be assigned to an empty candidate site (i.e., no emergency station opening). Economies of scale $(E o S)$ is considered in the capital cost, where parameter $K$ is the coefficient and $\alpha$ is the $E o S$ factor.

Moreover, function $F$ is the abstract form of decision rules that map from each sample scenario of uncertainty $\xi$ to a solution sequence (or vector) $x$. In other words, $F: \Xi \rightarrow X, X$ is the set of all possible solutions. The decision rules are described as "IF-THEN-ELSE" conditional-go statements and captured by non-anticipative constraints in the model, which differ from typical recourse decisions obtained in multi-stage stochastic programming. Typical solutions from multi-stage stochastic programming would dictate how much capacity to deploy based on uncertainty realizations (i.e. the recourse decisions), but without any consistent and constant guidelines for system operators to follow. In our proposed model, a decision rule regarding capacity expansion at site $j$ can be expressed as: IF a station at site $j$ missed $\beta_{j}^{1}$ number of emergency requests over a strategic period, THEN planners may consider deploying $\gamma_{j}^{1}$ units of capacity at this station until it reaches the maximal capacity; ELSE, do nothing. In the two sample scenarios $k$ and $l$ (for example), if the numbers of missed requests $\vartheta_{j k}^{t}$ and $\vartheta_{j l}^{t}$ are both greater than the threshold $\beta_{j}^{1}$, this station will then be expanded by $\gamma_{j}^{1}$ units of capacity in both scenarios.

\subsection{Modeling Assumptions}

The proposed framework is applied to a design problem focused on allocating emergency resources over the system's life cycle, subject to its KPIs. The objective of the design is to minimize the expected life cycle costs in the face of long term uncertainty, such as geo based incident arrival rate.

There are a few assumptions underlying the proposed model as applied to the EMS case under study. The response policy considered in the following case study (introduced in Section 5) is that emergency vehicle will only respond to incidents that occurred within the coverage area of the EMS station where the vehicle is stored and maintained. There are various possible response policies for EMS systems, and the proposed framework is independent from them, and the results will vary 
depending on the EMS system model. Here, one district can be covered by one and only one station. This indicates that incidents occurring in a given district can only be covered by emergency vehicles located in that district. One station can cover more than one district, however, so it is possible that the number of emergency stations is less than the number of districts. Moreover, only information observed up to a certain point can be used for making decisions (inherited from multi-stage programming), and such decisions are exactly the same if two cases follow the same scenario (i.e. non-anticipativity constraint accounted for in the model). The symbols and terminology used in these statements are introduced in Appendix 1, while the specific formulation of the multi-stage stochastic model with regards to the design problem discussed in Section 5 is presented in Appendix 2.

\section{Numerical Experiment}

\subsection{Problem Description}

In this section, the generic model developed in Section 4 is used to study a specific design problem regarding the capacity deployment of an EMS system through a numerical study. The study aims to answer the following research questions: 1) "How does the proposed approach to ROA based on decision rules compare with the results from standard methods in terms of objective function value and computational performance?" and 2) "Does the flexible design dominate stochastically rigid designs in terms of expected total cost?" The EMS system discussed in the numerical experiment is based on a hypothetical city, which could be thought of a small- or medium-sized one like many cities in the central and western regions of China or other developing and rapidly urbanizing countries (e.g., India). It may also be relevant to cities in more developed economies (e.g. Singapore) where EMS systems are in the planning stage, or undergoing significant changes in emergency incident patterns. Due to the population growth, the capacity of the system may need to be increased over time to achieve a desirable emergency request coverage rate or fleet size. A novel design based on the concept of flexibility is introduced as an important alternative to fulfill these requirements. This flexible design will be subsequently analyzed via a standard ROA tool and the proposed approach will be compared to typical rigid designs to reveal the value of the flexibility embedded in the system.

In reality in some countries (e.g., Singapore and China), an emergency vehicle is deployed at a candidate site that is reserved for this particular use, or temporarily stored at a rented public place (which is not considered in this paper). In this analysis, candidate sites and districts are simplified and geographically abstracted as nodes, as done by previous researchers (Beraldi and Bruni, 2009; Toregas et al., 1971). Also, based on conversations with a local EMS provider, strategic-level (e.g., capacity deployment) and tactical-level (e.g., vehicle (re-)allocation) decisions are made at different time horizons (i.e., asynchronous), as shown in Figure 4. It is assumed that a strategic period is about one year and contains four tactical periods. These assumptions can be modified according to different situations and contexts. 




Figure 4. An illustration of strategic and tactical planning horizons.

In this study, ten candidate sites and districts are considered for coverage by the EMS system. Table 1 lists the coordinate information for all candidate sites (or districts), which are adapted from the 36node test problem published by Batta et al. (1989). As mentioned above, the problem size considered is typical of cities in the middle or Western parts of China, where the issue of deploying and operating additional EMS capacity in the future is relevant. To consider the flexible design as an alternative and complementary design, it is assumed that there is an existing station with one unit of capacity at District 3. The incident arrival rate (i.e., number of emergency requests per hour) for each district is calibrated from actual publicly available data from Singapore in the year 2006 (Ong et al., 2009). Specifically, the incident rates are randomly generated based on a uniform distribution between 0.03794 and 0.75210 calibrated on historical data. These two values are $-50 \%$ of the lowest incident rate of a district (i.e., 0.07588 in Bras Basah/Bugis), and $+50 \%$ of the highest incident rate of a district (i.e., 0.48340 in Woodlands/Marsiling) in Singapore. The incident rate for a district is calculated by the following Equation (14)

$$
N_{j}=n_{j} \cdot H \cdot Q
$$

where $H$ is the number of hours per day and $Q$ is the number of days associated with the total number of incidents $N_{j}$ at site $j$. Symbol $n_{j}$ thus represents the incident rate at site $j$. The $-/+50 \%$ manipulation is used to broaden the range of possible incident rates because the population in this hypothetical city may not be distributed in the same manner as Singapore. While this study aims to rest on plausible historical data for demonstration purposes, it does not provide specific recommendations for any given country or city.

Table 1. The locations and incident rates of all ten candidate sites of the EMS system

\begin{tabular}{|l|c|c|l|c|c|}
\hline Districts (sites) & Location (x, y) & Incident rate & Districts (sites) & Location (x, y) & Incident rate \\
\hline 1 & $(32,31)$ & 0.04840 & 6 & $(27,29)$ & 0.50140 \\
\hline 2 & $(29,32)$ & 0.59620 & 7 & $(24,33)$ & 0.23390 \\
\hline
\end{tabular}




\begin{tabular}{|l|c|c|l|c|c|}
\hline 3 & $(27,36)$ & 0.58700 & 8 & $(34,30)$ & 0.64780 \\
\hline 4 & $(29,29)$ & 0.09690 & 9 & $(29,21)$ & 0.13360 \\
\hline 5 & $(32,29)$ & 0.09270 & 10 & $(33,28)$ & 0.64000 \\
\hline
\end{tabular}

For considerations of a long-term decision-making horizon, more sample incident rates are generated through a Geometric Brownian Motion (GBM) process (as found in Equation (15) based on (Ross, 2014). $W_{t}$ is a Wiener process or so-called Brownian motion, while $\mu$ and $\sigma$ are the expected growth rate and volatility based on historical data, respectively. $n_{j, t}$ denotes the uncertainty on candidate site $j$ at state $t$. It is reasonable to generate samples via GBM since it consists of a mean growth rate (the drift - based on the idea that population demographics will grow slowly and stably in the long term) plus a random shock (the volatility - which is normally distributed around the mean growth rate, to simulate the iid process, since each evolutionary scenario might differ).

$$
d n_{j, t}=\mu n_{j, t} d t+\sigma n_{j, t} d W_{t} .
$$

To assume better values for the expected growth rate and the volatility of incident requests, a statistical analysis is conducted considering historical annual EMS calls in two cities located in different countries. The analysis is similar as presented in Jin et al. (2011), which assumes that the logarithm of the percentage change for each year is normally distributed. Figure 5 shows the number of annual EMS calls in Singapore from 2000 to 2015 (Singapore Civil Defence Force, 2016), and in Boston from 2007 to 2015 (City of Boston, 2016). The expected growth rate (i.e., $\mu$ ) of incident requests for Singapore is $6.05 \%$, while for Boston it is $2.26 \%$. Regarding the volatility (i.e., $\sigma$ ), Singapore $(2.75 \%)$ is slightly higher than Boston (1.55\%). These observations may be caused by a population growing faster in Singapore than Boston, while realizing that the population growth in both cities is relatively stable in terms of volatility. However, it is possible that the population grows rapidly but that the growth is not stable due to population shifts. This is the case for many middle or Western cities in China for which historical data is scarce. Thus, when setting the assumptions for the base case, it is assumed that the value of expected growth rate is $5 \%$ and the value of volatility is $10 \%$. The sensitivity analysis performed in Section 5.3 tests the relative influence of these parameters on the study conclusions, which correspond to cities where the incident rates might grow slower or faster. 


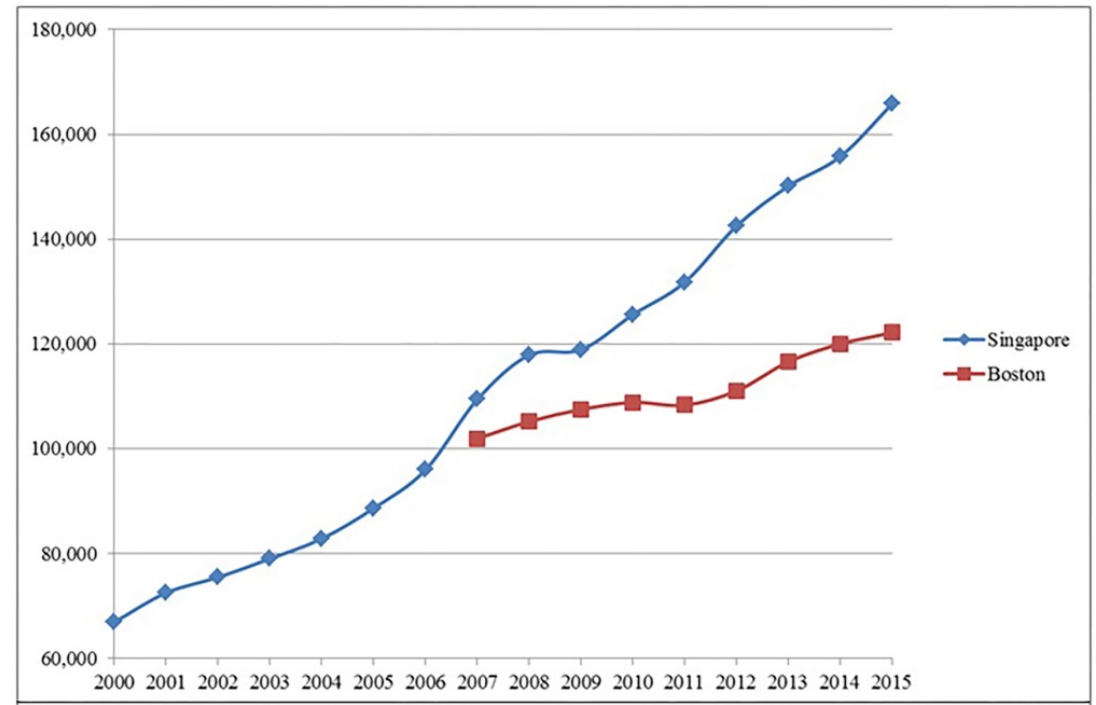

Figure 5. Number of annual EMS calls in Singapore (2000 to 2015), and Boston (2007 to 2015).

Figure 6 shows 1,000 scenario samples of incident rates generated based on the GBM process and the assumptions of the input parameters discussed above. The coverage of an emergency station is considered as a circle, and its radius is calculated using a Euclidean metric (which is ten units of the distance standard). Emergency vehicles can respond to emergency requests occurring within this coverage within the predetermined time (e.g., 11 minutes). In this case study, a maximum of four vehicles can be allocated to one station. All assumptions for the parameter details can be found in Table 7 in Appendix 3. Exact values cannot be disclosed due to a confidentiality agreement with a local EMS provider. The relative scale, however, captures the realities of system planning and operations, based on discussions with the local EMS provider (Local EMS Provider, 2012).

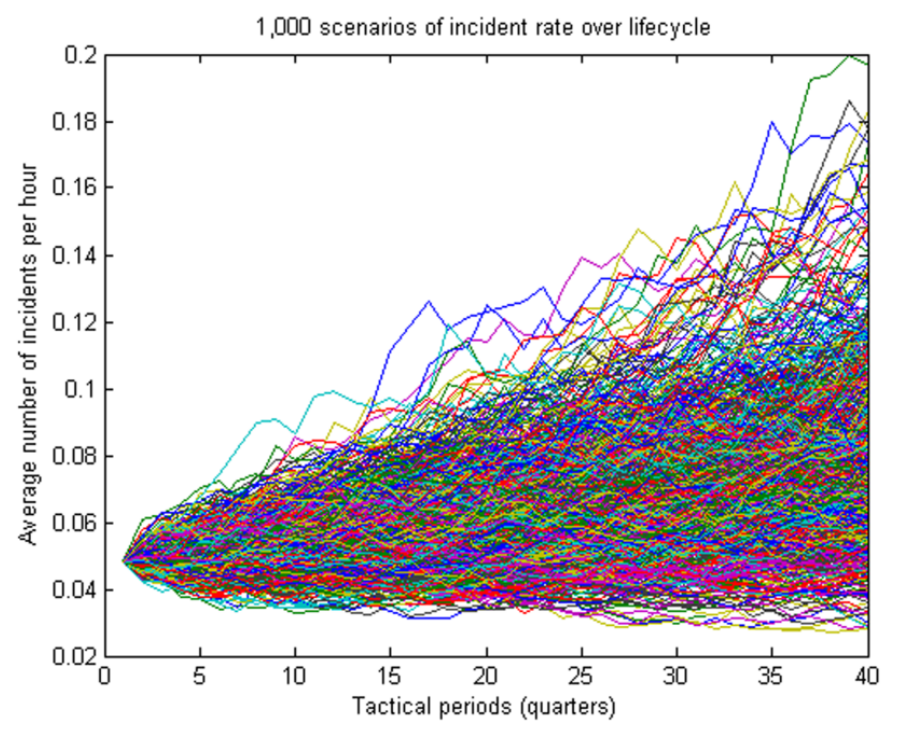

Figure 6. 1,000 samples of the incident rate in District No.1 over 40 years. 
The objective of this design problem is to minimize the expected total cost over the system life cycle, subject to the requirement of requested responsiveness. Other objective functions and/or main constraints can be set (e.g. maximize coverage subject to limited budget). The problem analyzed corresponds to the one facing a local EMS provider, based on personal communications. Such problem may differ in other cities and/or countries. The total cost consists of the capital cost (cost associated with deploying emergency stations and purchasing vehicles), operations and maintenance cost, and assignment cost. The expense of deploying stations is calculated accounting for $E o S$, as shown in Equation (16), which is an exact form of function $H($.$) . Parameter l$ denotes the units of capacity deployed at a site (which is no greater than 4), and $\alpha=0.9$ denotes the $E o S$ factor used in this example analysis. $\alpha$ is the parameter of the power function that models economies of scale. When $\alpha=1$, it effectively implies very weak economies of scale. In this case, $\alpha$ (tuneable) cannot be zero or even less than that (negative value). Its value typically lies between 0.6 and 1.0, depending on the industry (de Neufville, 2008). The assignment cost is proportional to the distance between a candidate site and a district, which accounts for the cost spent dispatching vehicles.

$$
c_{l}=c_{0} U_{l}^{\alpha}, l=1, \ldots, 4
$$

The value of flexibility is calculated via the following equation, which represents the difference between the expected total cost of a rigid design and the flexible design. The expected total cost is obtained through an out-of-sample analysis performed later. The cost associated with deploying flexible stations is usually higher than that of rigid stations. This cost premium for enabling flexibility, however, is assumed to be zero as it heavily depends on the local installation conditions and thus cannot be estimated well in this analysis. The value of flexibility is a non-negative value. The max[] condition captures the fact that, if flexibility does not create positive value (e.g., cost savings), it will not be considered - and, therefore, its lower bound is zero. Positive value indicates that the flexible design performs better than the rigid design. The value of flexibility determines the upper bound of the cost premium that planners should be willing to pay to enable the flexibility in the system (e.g. purchasing extra land, designing stations so the capacity of the infrastructure can be easily expanded).

$$
E[\operatorname{Value}(\text { Flex })]=\max \{E[\operatorname{Total}(\text { Rigid })]-E[\operatorname{Total}(\text { Flex })], 0\}
$$

The computational analysis was run on a desktop machine with a $3.30 \mathrm{GHz} \mathrm{CPU}$ and $8.0 \mathrm{~GB}$ of random access memory (RAM). Both the rigid and flexible designs were coded in AIMMS 3.14 (AIMMS, 2013) and CPLEX 12.6 was selected as the mixed integer programming (MIP) solver. The flexible design was also analyzed based on a dynamic programming model coded in Visual Studio 14, for the purpose of comparing the decision rule based solutions with the solutions based on standard ROA. 


\subsection{Solution Comparison}

Compared to a rigid design solution that deploys all capacity at once, a flexible design allows gradual deployment of capacity over time and space. To calculate the value of flexibility, standard ROA tools like multinomial lattice and ADP, and the proposed approach based on multi-stage stochastic programming (SP), are applied in the numerical experiment. The ADP is similar to the one used in (Cardin et al., 2017a), in which the objective function is approximated by a linear value function $\tilde{J}$ that can be formulated as

$$
\tilde{J}=\rho_{0}+\rho_{1} x_{1}+\rho_{2} d_{1}+\cdots+\rho_{2|I|-1} x_{|I|}+\rho_{2|I|} d_{|I|} .
$$

This function is a weighted combination of important quantities, such as the cost for installing stations, purchasing vehicles, etc. Parameter $|I|$ denotes the number of uncertainty drivers or the number of districts in this case. For simplicity, it is assumed that the number of districts is equal to the number of candidate sites (i.e., $|I|=|J|$ ). It is also assumed that, for the standard ROA approaches, the assignment cost is eliminated from the total cost. This is because this cost is incurred in tactical periods rather than strategic periods, and thus cannot be captured in the nodes of a lattice structure.

Table 2 presents the objective function values obtained for the three ROA approaches in millions of US dollars, based on the same assumptions and input parameters, with the corresponding computational times in seconds presented in brackets. The objective functions for the three approaches are the same (i.e. minimize the expected total costs of the EMS system including installation costs of stations, costs of purchasing vehicles, etc. over its life cycle).

Table 2. Solution comparison across three ROA approaches (\$ million) and computational time (sec)

\begin{tabular}{|l|c|c|c|}
\hline Approaches & $|I|=2$ & $|I|=3$ & $|I|=10$ \\
\hline Multinomial lattice & $4.904(655.16)$ & $-(-)$ & $-(-)$ \\
\hline ADP & $3.067(59.94)$ & $5.168(65.14)$ & $-(-)$ \\
\hline Multi-stage SP & $3.301(11.95)$ & $6.046(599.00)$ & $15.98(24,754.00)$ \\
\hline
\end{tabular}

Overall, Table 2 shows that the proposed approach based on multi-stage SP is applicable for dealing with larger scale problems in an acceptable amount of time, as compared to standard ROA based on multinomial lattice and ADP. The multinomial lattice analysis can only handle problems with up to $|I|$ $=2$ districts; the algorithm execution runs out of memory when the number of districts increases to three. In contrast, APD solves problems with $|I|=3$ districts in a shorter time compared to the 
proposed approach (65.14 sec vs. $599 \mathrm{sec}$, respectively). ADP's computational time increases drastically (more than 24 hours), however, for the case of $|I|=10$ districts, and also ultimately runs out of memory. The proposed approach, in contrast, can deal with a larger numbers of districts and shows feasible computational times. Although the solutions of ADP are smaller than those of the proposed approach, ADP does not consider the assignment cost, which accounts for a significant proportion of the total cost. Even if the assignment cost could be considered in ADP (which would be very difficult to implement), the computational time would still be very long due to a large number of circulates and judgments in the program. This ultimately makes the ADP approach less practical and competitive as compared to the proposed approach, which can be used to answer the first research question.

To answer the second research question, two rigid designs are introduced for comparison. The first rigid design is referred to as the "least flexible" design, i.e., it embeds some level of operational flexibility in terms of vehicle allocation. It deploys all capacity (i.e., emergency stations and vehicles) at once at the beginning of the time period, and reallocates vehicles accordingly over the system life cycle in an optimal manner. It is a robust solution in the sense that it provides the minimum expected lifecycle cost based on the selected scenarios for optimization, but is rigid in the sense that it follows a pre-determined and fixed capacity allocation plan. The second design solution is a multi-period extension of the work by Beraldi and Bruni (2009) in which stations and vehicles can be deployed over time. It is referred to as the "less flexible" design because the resources are deployed gradually based on a pre-determined plan, rather than based on the realization of uncertainty scenarios. The less flexible design also captures, to some extent, a robust design philosophy, finding the deployment path (albeit rigid) that can deal best with a wide range of possibilities, without requiring the system to change dynamically over time (Jugulum and Frey, 2007). Compared to rigid designs, the flexible design gradually deploys and allocates resources based on a plan incorporating the optimal decision rules. This third solution is more dynamic as it creates a capacity deployment plan that adapts to uncertainty realizations in the optimization process and in the out-of-sample analysis. The resulting capacity deployment and operations is different, based on the uncertainty scenario realization for incident patterns in different districts.

Table 3 shows the features of the three alternatives in terms of the scale of the problem and optimization results. The number of scenarios considered in the SP model based on sample average approximation is ten. The LP bound is the optimal solution of the linear relaxation of the original MIP problem, while the best solution is the optimal solution obtained for the original MIP problem. Linear relaxation means that the type of decision variables is relaxed from integers to continuous numbers for the same problem. Theoretically, Best LP bound $\leq$ Best solution, and the equal sign only holds when the optimal solution of the linear relaxation are all integers and/or binaries. Given that there is only a $1.2 \%$ difference between the best and the worst solutions (i.e., 15.79 vs. 15.98 ) and the stochastic 
nature of the optimization, one may take into account that all design solutions perform approximately the same in the optimization analysis. The gap between the best solution and the best linear programming (LP) bound, on the contrary, is much greater for the less flexible and flexible designs, as compared to the least flexible design. This may be caused by the default algorithm terminating when reaching the upper limit on the number of iterations, before converging to the true global optimal solution. The flexible solution also requires significantly more computation time compared to the least and less flexible solutions. This is because the number of decision variables and constraints increase along with increasing considerations of flexibility. When more flexibility is embedded into the model, more binary and/or integer variables are needed for capturing decision-making dynamics (e.g., whether or not such flexibility is actually enabled), and more conditional-go decision rules are required as non-anticipative constraints.

Table 3. Comparison of characteristics and results of three design alternatives $(|N|=10, \operatorname{CoV}=0.95)$

\begin{tabular}{|l|c|c|c|c|c|c|}
\hline Alternatives & $\begin{array}{c}\text { No. of } \\
\text { constraints }\end{array}$ & $\begin{array}{c}\text { No. of } \\
\text { integer } \\
\text { variables }\end{array}$ & $\begin{array}{c}\text { Best LP bound } \\
\text { (\$million) }\end{array}$ & $\begin{array}{c}\text { Best solution } \\
(\$ \text { \$million })\end{array}$ & $\begin{array}{c}\text { Gap } \\
(\%)\end{array}$ & $\begin{array}{c}\text { CPLEX } \\
\text { time (sec) }\end{array}$ \\
\hline Least flexible & 49,365 & 40,441 & 15.64 & 15.79 & 0.98 & $3,368.8$ \\
\hline Less flexible & 49,815 & 40,810 & 14.78 & 15.90 & 7.01 & 9031.4 \\
\hline Flexible & 69,175 & 53,846 & 14.61 & 15.98 & 9.39 & $24,754.0$ \\
\hline
\end{tabular}

Figure 7 illustrates how capacity is deployed for the three design solutions at two districts using the same input assumptions and one sample scenario as an example. As can be seen, the flexible design solution deploys no capacity at District 7 , while both the least and less flexible designs deploy one unit of capacity at District 7 . The less flexible design, however, defers deploying capacity until strategic period seven rather than the beginning of the life cycle, as does the least flexible design. For both the least and less flexible designs, once capacity is deployed it cannot be changed until the end of the life cycle. The flexible design, however, can change the capacity if needed and possible, which is shown in Figure 7, depicting the capacity deployment dynamics at District 10 . There, the flexible solution deploys one unit of capacity at the beginning of the time period, and then expands capacity by one unit at strategic period eight. The least and less flexible designs, in contrast, do not deploy any capacity at District 10 in this scenario (dashed lines for least and less flexible designs overlap). Also, the difference between the capacity deployment occurring for the less flexible design in District 7, and for the flexible design in the District 10 is that the one in District 7 will occur, no matter what sample scenario is used. It is a rigid deployment plan, although it is optimal from a stochastic standpoint. In 
contrast, the capacity deployment shown in District 10 for the flexible design occurs for the particular sample scenario used in the example, because the criteria for deploying the capacity (explained below) are satisfied for this particular scenario only. Using a different sample scenario in the example would lead to a different capacity deployment path for the flexible design solution. Also, each site is independent in terms of decision making, following the decision rule guidelines, while the objective function is measured at the system level. This means that every candidate site may adapt flexibly based on their own governing decision rules, as summarized below in Table 4.
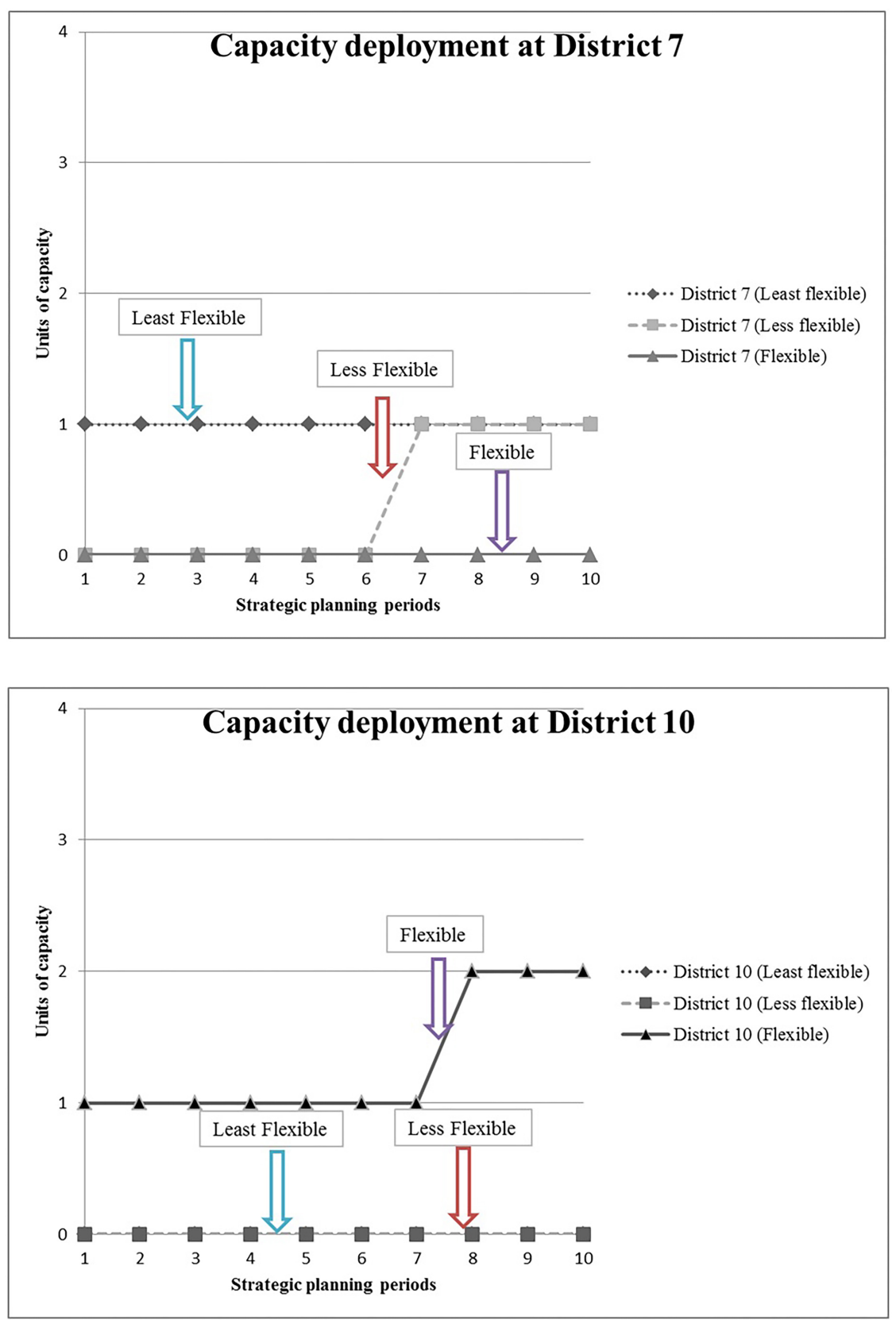

Figure 7. Visualization of capacity deployment of three design solutions for Districts 7 and 10.

Table 4 summarizes the optimal solution outputs of the flexible design for each district or site. In essence, it summarizes concisely the fully integrated guidelines for deploying the capacity in the 
example EMS system over time and space for the entire lifecycle, and for each district. Columns 2 to 5 list the corresponding values of the parameters that define the decision rules for each district. For example, to summarize the flexible solution for District 8 , the system operator should deploy initially $o_{8,1}^{1}=1$ unit of capacity in strategic period one $(S=1)$. If emergency vehicles at District 8 miss more than $\delta_{8}^{d}=50$ incident requests in a strategic period, the operators will expand $o_{8}^{u}=1$ unit of capacity at this site. This decision is revisited at each strategic period (which is why it leads to different deployment plans, based on how the incident patters evolve over time). If the operators did not install a station at strategic period one as recommended, but they want to do this at subsequent periods, then they should deploy $o_{8}^{o}=1$ unit of capacity if and only if the sum of the incident rates at District 8 is greater than or equal to $\delta_{8}^{o}=3.676$ (or equally, if the average incident rates is no less than 0.919). For other districts where $o_{j l}^{1}=0$, the initial capacity depends on the parameters of the decision rules. That is, if the sum of incident rates at district or site $j$ in a strategic period is no less than $\delta_{j}^{o}$, and there is no station available yet, then deploying $o_{j}^{o}$ units of capacity is recommended at this site. Note that there is an existing station at District 3 before the study life begins (i.e. before $t=$ 0 ), and thus the flexibility strategy is not applicable for District 3. Since the flexible solution produces capacity deployment paths that change depending on each uncertainty scenario realizations, it cannot be readily captured as a simple graph. Readers may consider the example capacity deployment paths for the flexible design at Districts 7 and 10 in Figure 7 as an illustration based on one uncertainty realization.

Table 4. Summary of the output of the flexible design solution.

\begin{tabular}{|l|c|c|c|c|c|}
\hline Districts (sites) & $\delta_{j}^{o}$ (incident rate) & $o_{j}^{o}$ (unit) & $\delta_{j}^{d}$ (No. incidents) & $o_{j}^{u}$ (unit) & $o_{j l}^{1}$ (unit) \\
\hline 1 & 0.486 & 1 & 50 & 1 & 0 \\
\hline 2 & 0.001 & 1 & 50 & 1 & $l=1$ \\
\hline 3 & - & - & - & - & - \\
\hline 4 & 0.793 & 1 & 50 & 1 & 0 \\
\hline 5 & 0.85 & 1 & 50 & 1 & 0 \\
\hline 6 & 3.319 & 1 & 50 & 1 & $l=1$ \\
\hline 7 & 2.492 & 1 & 50 & 1 & 0 \\
\hline 8 & 3.676 & 1 & 50 & 1 & $l=1$ \\
\hline
\end{tabular}




\begin{tabular}{|l|c|c|c|c|c|}
\hline 9 & 1.106 & 1 & 50 & 1 & 0 \\
\hline 10 & 0.001 & 1 & 50 & 1 & $l=1$ \\
\hline
\end{tabular}

An important step in evaluating how each solution performs in scenarios not used during optimization is to run an out-of-sample analysis. In statistics, such analysis aims to address the issue of over fitting, since solutions that rely only on in-sample tests are likely to understate the error, and over fit the solution features based on the data used in the optimization process (Tashman, 2000). Here, a large number of samples (i.e., 1,000) is used in the out-of-sample analysis; these samples are different from those used in the optimization in terms of their values. Samples used in in-sample optimization and out-of-sample analysis are generated by the same GBM process following the same distribution and parameters. In the out-of-sample analysis, however, no optimization is performed. The goal is to see how the solutions obtained from in-sample optimization perform with unforeseen scenarios. Therefore, the analysis provides a better assessment of the relative performance of each design solution than in the optimization analysis (summarized in Table 3).

Table 5 shows that the flexible design solution performs significantly better than other solutions in the out-of-sample analysis. It indicates that the flexible system is more adaptable to unforeseen scenarios - which is one of the purposes of enabling flexibility in the first place. The expected total cost of the flexible design is nearly $5 \%$ lower than that of the least flexible design, and about $1 \%$ lower than that of the less flexible design. The flexible design solution is also best in terms of P5 ( $5^{\text {th }}$ percentile, a measure of best case outcomes) and P95 ( $95^{\text {th }}$ percentile, a measure of worst case outcomes). Figure 8 shows the cumulative density functions for the three design solutions in the out-of-sample analysis, which provides a graphical representation of the results, leading to the same conclusions.

Table 5. Comparison of characteristics and results of the out-of-sample analysis $(|N|=1,000)$

\begin{tabular}{|l|c|c|c|c|c|c|c|}
\hline Alternatives & $\begin{array}{c}\text { No. } \\
\text { constraints }\end{array}$ & $\begin{array}{c}\text { No. } \\
\text { integer } \\
\text { var }\end{array}$ & $\begin{array}{c}\text { Mean } \\
(\text { \$million })\end{array}$ & $\begin{array}{c}\text { STD } \\
(\$ \text { \$million })\end{array}$ & $\begin{array}{c}\text { P5 } \\
(\text { \$million })\end{array}$ & $\begin{array}{c}\text { P95 } \\
(\text { \$million })\end{array}$ & $\begin{array}{c}\text { Total } \\
\text { time } \\
(\mathrm{sec})\end{array}$ \\
\hline Least flexible & 4,082 & 4,000 & 15.53 & 0.78 & 14.74 & 17.03 & 866.40 \\
\hline Less flexible & 4,802 & 4,000 & 14.91 & 0.99 & 13.95 & 16.74 & $1,184.70$ \\
\hline Flexible & 6,179 & 4,972 & 14.77 & 1.02 & 13.06 & 16.49 & $2,470.40$ \\
\hline Best? & - & - & Flexible & Least & Flexible & Flexible & - \\
\hline
\end{tabular}




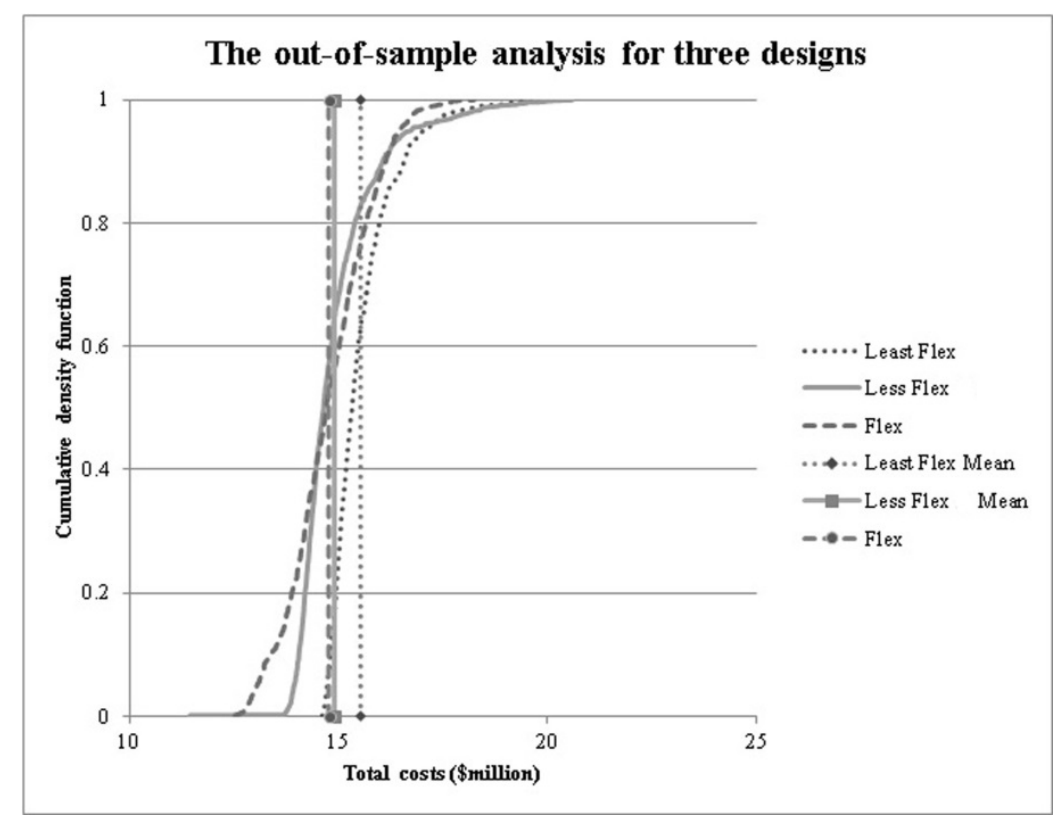

Figure 8. Cumulative distribution functions of the out-of-sample analysis.

\subsection{Sensitivity Analysis}

The sensitivity analysis next evaluates how solution outputs are affected by variability in input parameter assumptions. An adaptive One-Factor-at-A-Time (OFAT) approach (see Frey and Wang (2006)) is used to identify the significance of the different input parameters. Table 6 shows the values of various input parameters at three levels - low, base, and high. The low and high values equal the base value $-/+50 \%$, excluding the incident coverage rate. This is because such variation makes little sense when $\mathrm{CoV} \leq 0.8$. The low and high values are representative of countries where urbanization and incident rate growth may be slower or higher than for the base case. They also account for the different risk premiums associated with raising capital in different countries, as captured by different discount rates.

Figure 9 shows the results of the sensitivity analysis in terms of expected value of flexibility $(V o F)$. As defined in Equation (17), this value is the difference between the expected total cost of a rigid design (either least or less) and the flexible design. It can be seen that the $V o F$ is mostly affected by the expected growth rate, then by the volatility and $\mathrm{CoV}$. The faster the incident rates grows, the more valuable flexibility is in the face of growing uncertainty. As volatility increases, flexibility is also worth more as it enables the system to better adapt to changing conditions. At the other end of the spectrum, little volatility brings down the value of flexibility, since there is no need for change. Interestingly, the results show that flexibility is more valuable when the coverage requirement is higher. When comparing the flexible to the least flexible designs, the value of flexibility increases with an increasing discount rate because a higher discount rate provides more incentives to defer capacity deployment to a later time - thereby making more use of the flexibility. The impact of the 
discount rate is reduced when comparing the flexible to the less flexible design, since capacity is deployed over time in both cases.

Table 6 . Values of the input parameters in the sensitivity analysis

\begin{tabular}{|l|l|l|l|}
\hline & Low & Base & High \\
\hline Incident Coverage Rate $(\mathrm{CoV})$ & 0.90 & 0.95 & 0.98 \\
\hline Coverage radius & 5 & 10 & 15 \\
\hline Discount rate $(r)$ & $6 \%$ & $12 \%$ & $18 \%$ \\
\hline Expected growth rate $(\mu)$ & $2.5 \%$ & $5 \%$ & $7.5 \%$ \\
\hline Volatility $(\sigma)$ & $5 \%$ & $10 \%$ & $15 \%$ \\
\hline
\end{tabular}

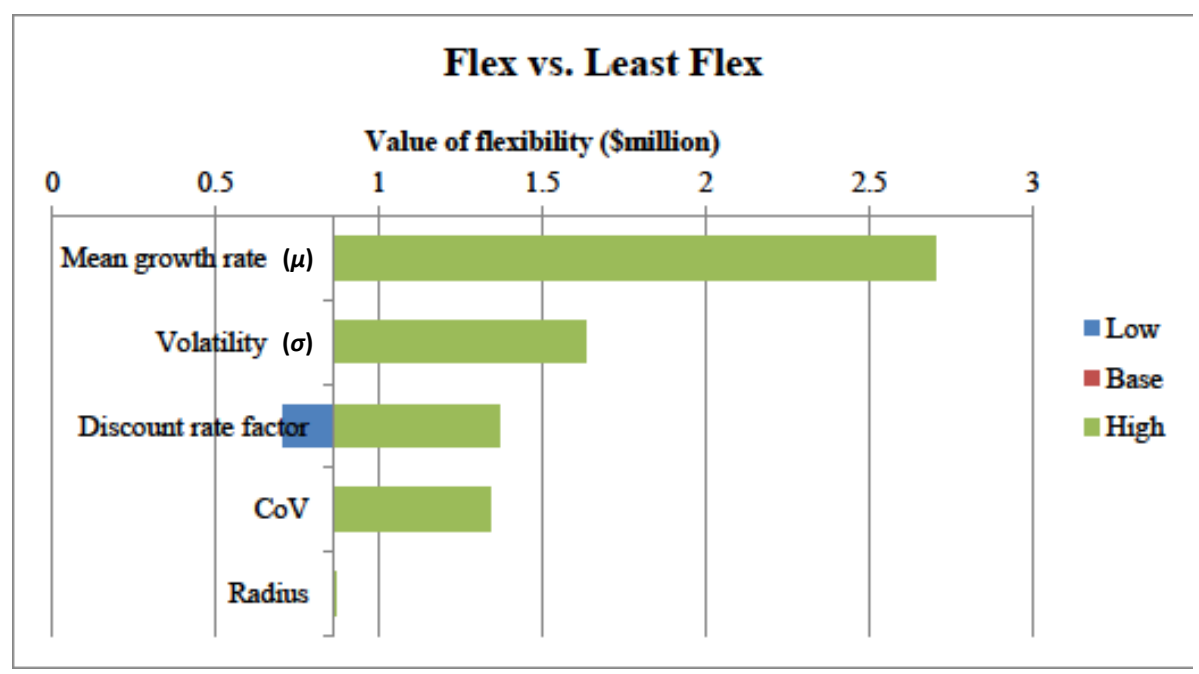




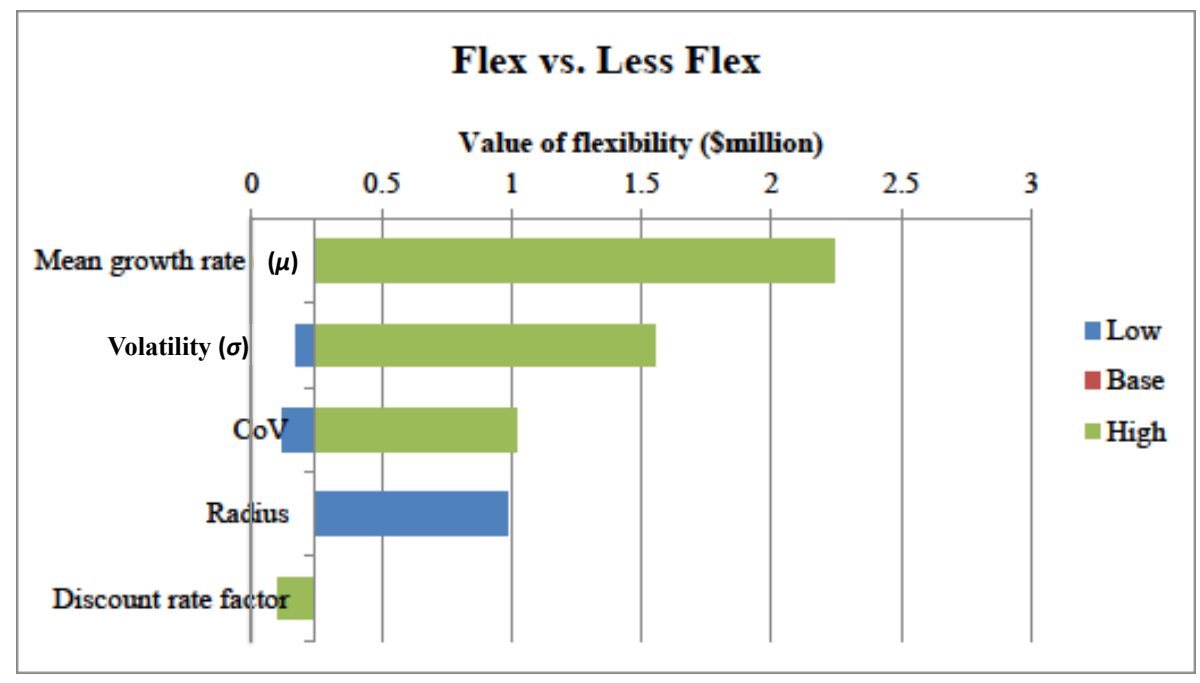

Figure 9. Tornado charts for the value of flexibility.

The above optimization and out-of-sample analysis are based on assumptions of the base value for the incident coverage rate. There is, however, a clear tradeoff between coverage rate and budget (e.g., an unlimited budget would converge to $100 \%$ coverage, but also stations and vehicles everywhere, which is not desirable). The complete numerical analysis provides a comparison between three designs under different coverage rates. Figure 10 represents the outcomes of the comparison in the form of a Pareto analysis, where the coverage rate and the expected total cost are the $\mathrm{x}$ - and $\mathrm{y}$-axis, respectively. Each dot indicates the average total cost correlating to a specific design and coverage rate. The curve shows the corresponding shape of the total cost for each design alternative.

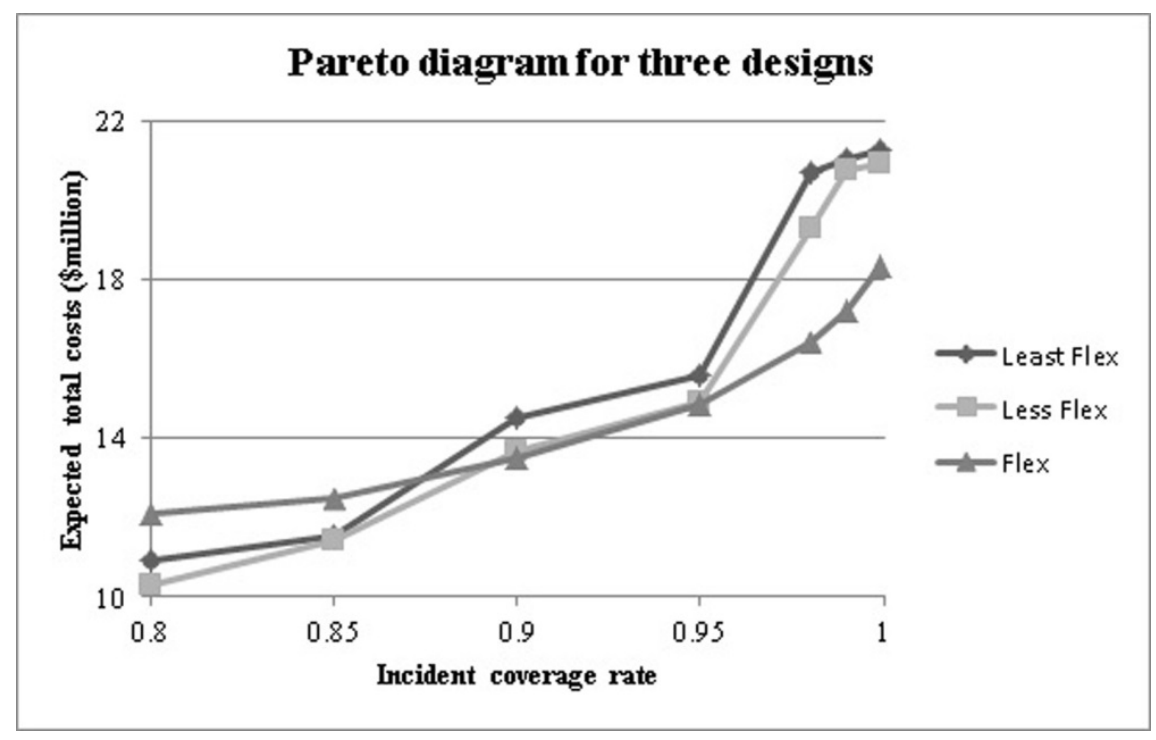

Figure 10. Comparison of three designs under different coverage rates.

The Pareto analysis shows that the flexible design dominates the other two designs when the coverage rate is required to be greater than 0.9 , confirming the observation from the sensitivity analysis. It also 
indicates that the flexible design performs much better than the more rigid designs when the coverage rate is considerably high (i.e., from 0.95 to 0.99 ). To achieve such a high-level coverage rate, one must deploy more than one unit of capacity at different sites (i.e., the least flexible design), or deploy capacity over time and space (i.e., the less flexible and flexible designs). The flexible design performs better than others because it deploys capacity based on uncertainty realizations rather than predetermined fixed plans. In contrast, the flexible design performs worst when the coverage rate is less than 0.88 . Since the decision rules are exercised once the conditions are satisfied, this assumption makes the flexible design more expensive when the system requires low coverage, as the design solution may deploy more capacity at some sites. In addition, one may expect that the least and less flexible designs would fail to meet the coverage requirement if the designs face extreme cases that are not considered in the optimization. These cases are handled better by the flexible design, as clearly demonstrated in the out-of-sample analysis.

\section{Discussion and Conclusions}

Improving the long-term life cycle performance of EMS systems is challenging. Growing economic development and rapid urbanization - especially in emerging countries - will inevitably lead city planners to face such issues in the future. This paper introduces a novel approach to EMS infrastructure systems design, capacity planning, and operations management accounting for strategiclevel flexibility and real options based on the notion of decision rules. This paper shows that this approach can improve the expected cost performance in the face of changing incident rates in the medium to long term using a case study based on actual historical incident data. This modeling framework performs real options and flexibility analysis based on decision rules for complex infrastructure systems using multi-stage stochastic programming. The approach supports further strategic and tactical implementation under uncertainty by aiming to mimic real-world design and decision-making, and providing EMS providers with solutions that are readily usable in operations. Compared to standard ROA approaches, the proposed framework is capable of dealing with a larger scale multi-facility deployment problems, while accounting for multiple flexibility strategies (i.e., phasing initial capacity deployment, on-site capacity expansion) in an acceptable amount of computation time. The out-of-sample analysis shows that the flexible design can reduce the expected total cost and even dominate more rigid solutions when the coverage rate required is at a high-level (e.g., $95 \%$ or higher), which is a desirable attribute in most cities. The sensitivity analysis shows that the most influential parameters for the value of flexibility are the expected growth rate, volatility, and coverage rate, in decreasing order of importance. It is demonstrated that the flexible design is better at taking advantage of upside opportunities and reducing downside risks, thus improving overall expected performance. The complete analysis shows that flexibility is a valuable approach to EMS system design, planning and operations, leading to potentially better solutions than rigid (or robust) solutions found in the literature, and currently used in practice. 
The proposed approach is practical, based on three perspectives: quality of the solution, computational time to the best solution, and ease of use of the resulting solutions. Firstly, as can be seen in Subsection 5.2 (Table 2), current standard approaches based on multinomial lattice and ADP cannot deal with large scale problems in the context of multi-facility capacity deployment. In other words, standard approaches may not be well suited in for real-world applications since they cannot provide adequate solutions to the problem considered. As an alternative, our proposed approach can handle middle to large scale problems, and the solution is acceptable as compared to the theoretical optimal solution. Secondly, the computation time of our proposed approach is not overly long for practical applications. Although the computational complexity of ADP is polynomial $\left(O\left(n^{i}\right)\right)$ and much shorter than the proposed approach, it cannot handle large scale problems, as seen in Table 2. The proposed approach, however, enables solutions using a hybrid heuristics (e.g., Bender's decomposition plus branch and bound) within a considerably short time, while considering a larger scale problem. Showing the development of such heuristics, however, is out of the scope of this paper, but interested readers may refer to the work by Zhang (2016) for further details. Thirdly, the solutions obtained based on the decision rule methodology are readily usable by decision-makers in operations, in the form of stochastically optimal IF-THEN-ELSE statements. Hence, the proposed guidelines can be applied directly during operations, and the solution does not need to be re-evaluated at each time period (or stage), as is the case for the multinomial lattice and ADP approaches.

There are several shortcomings in the analysis presented here that can fuel opportunities for future work. Firstly, the model inherits typical weaknesses of a stochastic programming approach, where a sample average approximation is used. The observed performance differs significantly when outsample scenarios are used, even though the flexible solution is shown to handle such cases better than rigid designs. Secondly, the decision rules regarding phasing deployment depend only on uncertainty realizations (i.e., incident rates). Moreover, computational time is always a concern for multi-facility resource allocation problems. The size of the problem increases significantly in terms of constraints and integer variables when more districts and sample scenarios are considered (e.g., $|I| \geq 20$ and/or $|N| \geq 15)$. The model then becomes unsolvable within a reasonable time.

Regarding opportunities for future work and improvement, a heuristic or an exact-heuristic hybrid algorithm may be useful for solving the problem within a reasonable time and reaching a good enough solution. Work along this line was introduced by (Zhang, 2016). Besides, the proposed approach could be modified to analyze other strategic- and/or tactical-level flexibility strategies (e.g., temporary or permanent station closures, periodic vehicle rotations) or combinations thereof, an EMS system that is larger and/or of larger complexity, and/or to test various forms of decision rules. Indeed, changing the decision rules may not greatly affect the algorithm based on the current model. Other objective functions and/or constraints could be studied following a similar methodological approach. A generalization of the proposed model could apply to the design of flexible EMS systems, or to more 
general multi-facility engineering systems, as done in (Cardin et al., 2017b). The issues of determining whether it is feasible or when it is optimal to exercise the flexibilities in real-world operations and from an implementation standpoint are also important, and should be addressed in future work, such as done in (Cardin et al., 2015a).

Acknowledgment

References

Acciaro, M., 2014. Real option analysis for environmental compliance: LNG and emission control areas. Transportation Research Part D: Transport and Environment 28, 41-50.

AIMMS, 2013. 3.14 ed. Paragon Decision Technology B.V., The Netherlands.

An, S., Cui, N., Bai, Y., Xie, W., Chen, M., Ouyang, Y., 2015. Reliable emergency service facility location under facility disruption, en-route congestion and in-facility queuing. Transportation Research Part E: Logistics and Transportation Review 82, 199-216.

Ball, M.O., Lin, F.L., 1993. A Reliability Model Applied to Emergency Service Vehicle Location. Operations Research 41(1), 18-36.

Baron, O., Berman, O., Kim, S., Krass, D., 2009. Ensuring Feasibility in Location Problems with Stochastic Demands and Congestion. IIE Transactions 41(5), 467-481.

Başar, A., Çatay, B., Ünlüyurt, T., 2011. A Multi-period Double Coverage Approach for Locating the Emergency Medical Service Stations in Istanbul. Journal of the Operational Research Society 62(4), 627-637.

Başar, A., Çatay, B., Ünlüyurt, T., 2012. A Taxonomy for Emergency Service Station Location Problem. Optimization Letters 6(6), 1147-1160.

Batta, R., Dolan, J.M., Krishnamurthy, N.N., 1989. The Maximal Expected Covering Location Problem: Revisited. Transportation Science 23(4), 277-287.

Bellman, R., 1952. On the Theory of Dynamic Programming, Proceedings of the Proceedings of the National Academy of Sciences of the United States of America, City, pp. 716-719.

Beraldi, P., Bruni, M., 2009. A Probabilistic Model Applied to Emergency Service Vehicle Location. European Journal of Operational Research 196(1), 323-331.

Berman, O., Hajizadeh, I., Krass, D., 2013. The Maximum Covering Problem with Travel Time Uncertainty. IIE Transactions 43(1), 81-96. 
Cardin, M.-A., 2014. Enabling Flexibility in Engineering Systems: A Taxonomy of Procedures and a Design Framework. Journal of Mechanical Design 136(1), 011005-011005-14.

Cardin, M.-A., Hu, J., 2016. Analyzing the Tradeoffs Between Economies of Scale, Time-Value of Money, and Flexibility in Design Under Uncertainty: Study of Centralized vs. Decentralized Wasteto-Energy Systems. Journal of Mechanical Design 138(1), 011401-011401-11.

Cardin, M.-A., Jiang, Y., Yue, H.K.H., Fu, H., 2015a. Training Design and Management of Flexible Engineering Systems: An Empirical Study Using Simulation Games. IEEE Transactions on Systems, Man, and Cybernetics: Systems 45(9), 1268-1280.

Cardin, M.-A., Ranjbar Bourani, M., de Neufville, R., 2015b. Improving the Lifecycle Performance of Engineering Projects with Flexible Strategies: Example of On-Shore LNG Production Design. Systems Engineering 18(3), 253-268.

Cardin, M.-A., Xie, Q., Ng, T.S., Wang, S., Hu, J., 2017a. An approach for analyzing and managing flexibility in engineering systems design based on decision rules and multistage stochastic programming. IISE Transactions 49(1), 1-12.

Cardin, M.-A., Zhang, S., Nuttall, W.J., 2017b. Strategic Real Option and Flexibility Analysis for Nuclear Power Plants Considering Uncertainty in Electricity Demand and Public Acceptance. Energy Economics 64, 226-237.

Chen, A.Y., Yu, T.-Y., 2016. Network based temporary facility location for the Emergency Medical Services considering the disaster induced demand and the transportation infrastructure in disaster response. Transportation Research Part B: Methodological 91, 408-423.

Chow, J.Y.J., Regan, A.C., 2011a. Network-based real option models. Transportation Research Part B: Methodological 45(4), 682-695.

Chow, J.Y.J., Regan, A.C., 2011b. Real Option Pricing of Network Design Investments. Transportation Science 45(1), 50-63.

Chow, J.Y.J., Regan, A.C., 2011c. Resource Location and Relocation Models with Rolling Horizon Forecasting for Wildland Fire Planning. INFOR: Information Systems and Operational Research 49(1), 31-43.

Chow, J.Y.J., Regan, A.C., Ranaiefar, F., Arkhipov, D.I., 2011. A network option portfolio management framework for adaptive transportation planning. Transportation Research Part A: Policy and Practice 45(8), 765-778. 
Chow, J.Y.J., Sayarshad, H.R., 2016. Reference Policies for Non-myopic Sequential Network Design and Timing Problems. Networks and Spatial Economics 16(4), 1183-1209.

Church, R., ReVelle, C., 1974. The Maximal Covering Location Problem. Papers in Regional Science: The Journal of the RSAI 32(1), 101-118.

City of Boston, 2016. Emergency Medical Services.

Copeland, T.E., Antikarov, V., 2001. Real Options: A Practitioner's Guide. Thomson Texere, New York, NY.

Daskin, M.S., 1983. A Maximum Expected Covering Location Model: Formulation, Properties and Heuristic solution. Transportation Science 17(1), 48-70.

de Neufville, R., 2008. Lecture Notes, ESD.71: Engineering Systems Analysis for Design. Massachusetts Institute of Technology, Cambridge, MA, United States.

de Neufville, R., Scholtes, S., 2011. Flexibility in Engineering Design. MIT Press, Cambridge, MA, United States.

de Neufville, R., Scholtes, S., Wang, T., 2006. Real Options by Spreadsheet: Parking Garage Case Example. Journal of Infrastructure Systems 12(2), 107-111.

Dixit, A.K., Pindyck, R.S., 1994. Investment under Uncertainty. Princeton University Press, Princeton, New Jersey, USA.

Farahani, R.Z., Asgari, N., Heidari, N., Hosseininia, M., Goh, M., 2012. Covering Problems in Facility Location: A Review. Computers \& Industrial Engineering 62(1), 368-407.

Farahani, R.Z., Drezner, Z., Asgari, N., 2009. Single Facility Location and Relocation Problem with Time Dependent Weights and Discrete Planning Horizon. Annals of Operations Research 167(1), 353-368.

Frey, D.D., Wang, H., 2006. Adaptive One-Factor-at-a-Time Experimentation and Expected Value of Improvement. Technometrics 48(3), 418-431.

Ghaderi, A., Jabalameli, M.S., 2013. Modeling the Budget-constrained Dynamic Uncapacitated Facility Location - network Design Problem and Solving it via Two Efficient Heuristics: A Case Study of Health Care. Mathematical and Computer Modelling 57(3-4), 382-400. 
Gunawardane, G., 1982. Dynamic Versions of Set Covering Type Public Facility Location Problems. European Journal of Operational Research 10(2), 190-195.

Hung, K.K.C., Cheung, C.S.K., Rainer, T.H., Graham, C.A., 2009. EMS systems in China. Resuscitation 80(7), 732-735.

Iannoni, A.P., Chiyoshi, F., Morabito, R., 2015. A spatially distributed queuing model considering dispatching policies with server reservation. Transportation Research Part E: Logistics and Transportation Review 75, 49-66.

Iannoni, A.P., Morabito, R., 2007. A multiple dispatch and partial backup hypercube queuing model to analyze emergency medical systems on highways. Transportation Research Part E: Logistics and Transportation Review 43(6), 755-771.

Jin, S., Ryan, S.M., Watson, J.-P., Woodruff, D.L., 2011. Modeling and solving a large-scale generation expansion planning problem under uncertainty. Energy Systems 2(3), 209-242.

Jugulum, R., Frey, D.D., 2007. Toward a Taxonomy of Concept Designs for Improved Robustness. Journal of Engineering Design 18(2), 139-156.

Khansa, L., Liginlal, D., 2009. Valuing the flexibility of investing in security process innovations. European Journal of Operational Research 192(1), 216-235.

Kort, P.M., Murto, P., Pawlina, G., 2010. Uncertainty and stepwise investment. European Journal of Operational Research 202(1), 196-203.

Lagoudakis, M.G., Parr, R., 2003. Least-Squares Policy Iteration. Journal of Machine Learning Research 4(Dec), 1107-1149.

Li, X., Zhao, Z., Zhu, X., Wyatt, T., 2011. Covering models and optimization techniques for emergency response facility location and planning: A review. Mathematical Methods of Operations Research 74(3), 281-310.

Liu, Y., Li, Z., Liu, J., Patel, H., 2016. A double standard model for allocating limited emergency medical service vehicle resources ensuring service reliability. Transportation Research Part C: Emerging Technologies 69, 120-133.

Local EMS Provider, 2012. Personal Communications with local EMS provider, Singapore.

Luo, J., 2015. A simulation-based method to evaluate the impact of product architecture on product evolvability. Research in Engineering Design 26(4), 355-371. 
McLay, L.A., 2009. A Maximum Expected Covering Location Model with Two Types of Servers. IIE Transactions 41(8), 730-741.

Myers, S.C., 1984. Finance Theory and Financial Strategy. Interfaces 14(1), 126-137.

Nair, R., Miller-Hooks, E., 2009. Evaluation of Relocation Strategies for Emergency Medical Service Vehicles. Transportation Research Record: Journal of the Transportation Research Board 2137, 6373.

Ong, M.E., Ng, F.S., Overton, J., Yap, S., Andresen, D., Yong, D.K., Lim, S.H., Anantharaman, V., 2009. Geographic-time distribution of ambulance calls in Singapore: utility of geographic information system in ambulance deployment (CARE 3). Annals of the Academy of Medicine, Singapore 38(3), 184-191.

Powell, W.B., 2009. What you should know about approximate dynamic programming. Naval Research Logistics 56(3), 239-249.

Powell, W.B., Simao, H.P., Bouzaiene-Ayari, B., 2012. Approximate dynamic programming in transportation and logistics: a unified framework. EURO Journal on Transportation and Logistics $1(3), 237-284$.

Rau, P., Spinler, S., 2016. Investment into container shipping capacity: A real options approach in oligopolistic competition. Transportation Research Part E: Logistics and Transportation Review 93, 130-147.

Ross, S.M., 2014. Introduction to Probability Models. Elsevier, Amsterdam.

Ruszczyński, A., Shapiro, A., 2003. Stochastic Programming In: Ruszczyński, A., Shaprio, A. (Eds.), Handbook in Operations Research and Management Science. Elsevier.

Saleh, J.H., Mark, G., Jordan, N.C., 2009. Flexibility: A Multi-Disciplinary Literature Review and a Research Agenda for Designing Flexible Engineering Systems. Journal of Engineering Design 20(3), 307-323.

Savage, S., 2002. The Flaw of Averages. Harvard Business Review 4.

Shapiro, A., 2013. Sample Average Approximation, In: Gass, S.I., Fu, M.C. (Eds.), Encyclopedia of Operations Research and Management Science. Springer US, Boston, MA, pp. 1350-1355.

Sharma, M., Brandler, E.S., 2014. Emergency Medical Services in India: The Present and Future. Prehospital and Disaster Medicine 29(3), 307-310. 
Shishebori, D., Yousefi Babadi, A., 2015. Robust and reliable medical services network design under uncertain environment and system disruptions. Transportation Research Part E: Logistics and Transportation Review 77, 268-288.

Singapore Civil Defence Force, 2016. General Statistics.

Tashman, L.J., 2000. Out-of-sample tests of forecasting accuracy: an analysis and review. International Journal of Forecasting 16(4), 437-450.

Toregas, C., Swain, R., ReVelle, C., Bergman, L., 1971. The Location of Emergency Service Facilities. Operations Research 19(6), 1363-1373.

Trigeorgis, L., 1996. Real Options: Managerial Flexibility and Strategy in resource Allocation. MIT Press, Cambridge, MA, United States.

Wang, T., de Neufville, R., 2005. Real Options “in” Projects, 9th Real Options Annual International Conference, Paris, France.

Zhang, S., 2016. A Stochastic Programming Approach to Analyze Design and Management of Flexibility in Infrastructure Systems Operating Under Long-Term Uncertainty, Department of Industrial and Systems Engineering. National University of Singapore. 


\section{Supplementary Material}

Appendix 1. Nomenclature

\section{Symbols and Terminology}

$S=$ the set of strategic periods of an entire life cycle $(s \in S)$;

$S_{1}=$ the set of strategic periods where $s=1$;

$S_{2}=$ the set of strategic periods where $s \geq 2$;

$S_{3}=$ the set of strategic periods exluding $s=|S|$;

$T=$ the set of tactical periods $(t \in T)$, partitioned equally into $|S|$ subsets, noted as $T_{S}$;

$T_{1}=$ the set of strategic periods where $t=1$;

$E_{S}=$ the length of a strategic period. It may range from one to several years;

$E_{t}=$ the length of a tactical period. It may range from several weeks to several months;

$E=$ the length of review periods $\left(e \in E\right.$ ). The maximal length is denoted as $a_{m}$, which is equal to $\left\lfloor E_{s} / E_{t}\right\rfloor$

$I=$ the set of districts to be covered in the system $(i \in I)$;

$J=$ the set of candidate sites for allocating stations $(j \in J)$;

$N=$ the set of emergency incident scenarios $(n \in N)$;

$L=$ the set of station phases $(l \in L)$. The corresponding capacity, installation cost, and operation cost of a phase $l$ station are denoted as $U_{l}, c_{l}$ and $m_{l}$, respectively;

$L_{1}=$ the set of station phases with the minimum capacity (i.e., $U_{l}=1$ );

$L_{2}=$ the set of station phases with the maximum capacity (i.e., $U_{l}=|L|$ );

$L_{3}=$ the set of station phases without the maximum capacity (i.e., $U_{l}<|L|$ );

$N_{i}=$ the set of candidate sites that can cover district $i$. That is, ambulances allocated to site $j \in N_{i}$ can respond to incidents in $i$ within a predetermined time (e.g., 11 minutes), usually to satisfy the EMS provider's required key performance indicator (KPI) (e.g., serve a given percentage of calls within a given time); 
$M_{j}=$ the set of districts that can be covered by candidate site $j$. The number of elements in this set is denoted as $\left|M_{j}\right|$;

$d_{i t n}=$ the average number of emergency incidents per hour (i.e., the incident arrival rate) in district $i$ within tactical period $t$ under scenario $n$;

$c_{v}, m_{v}=$ the unit cost of a medical vehicle (i.e., cost for purchasing an emergency vehicle) and its corresponding maintenance cost;

$c_{u}=$ the unit cost per phase for capacity expansion;

$q_{i j}=$ the corresponding cost of assigning district $i$ to site $j$;

$\mathrm{CoV}=$ the required incident coverage rate;

$r_{t}, r_{s}=$ the discount rate for tactical period $t$ and strategic period $s$, respectively;

$p_{n}=$ the corresponding probability of scenario $n$;

$h=$ the number of hours in a tactical period;

$\varepsilon, M=$ a small tolerance and an arbitrary large integer, used to ensure a given constraint is always or never satisfied.

\section{Variables (initial configuration)}

$o_{j l}^{1}=1$ if a phase $l$ station is deployed at site $j$ when strategic period $s \in S_{1}$, and zero otherwise;

$v^{1}=$ the number of vehicles purchased when strategic period $s \in S_{1}$

\section{Variables (decision rules)}

$o_{j}^{o}=$ the capacity to be deployed at site $j$ if phasing deployment is exercised;

$o_{j}^{u}=$ the capacity to be deployed at site $j$ if on-site expansion is exercised;

$\delta_{j}^{o}=$ the incident rate for exercising phased deployment at site $j$ when strategic period $s \in S_{2}$;

$\delta_{j}^{d}=$ the number of missed incidents for exercising on-site capacity expansion at site $j$ when strategic period $s \in S_{2}$ 


\section{Auxiliary variables}

$o_{j l s n}^{2}=1$ if a phase $l$ station is opened at site $j$ when strategic period $s \in S_{2}$ under scenario $n$, and zero otherwise;

$x_{j l s n}=1$ if there is a station at site $j$ at strategic period $s$ under scenario $n$ that has at least phase $l$ capacity, and zero otherwise;

$u_{j l s n}=1$ if the station at site $j$ is expanded $l$ unit when strategic period $s \in S_{2}$ under scenario $n$, and zero otherwise;

$y_{i j t n}=1$ if district $i$ is assigned to site $j$ at tactical period $t$ under scenario $n$, and zero otherwise;

$v_{s n}^{2}=$ the number of vehicles purchased when strategic period $s \in S_{2}$ under scenario $n$;

$w_{j t n}=$ the number of vehicles allocated at site $j$ at tactical period $t$ under scenario $n$;

$\omega_{j t n}, \xi_{j t n}=$ non-negative variables, used to express absolute values of other variables. 
Appendix 2. Mathematical Model

$$
\begin{gathered}
\min \sum_{n} p_{n}\left[\sum_{s}\left(r_{s} \sum_{j} \sum_{l}\left(c_{l} o_{j l s n}^{2}+c_{u} u_{j l s n}\right)+r_{s} c_{v} v_{s n}^{2}+\sum_{t \in T_{s}} m_{l} r_{t} w_{j t n}\right)\right. \\
\left.+\sum_{t}\left(\sum_{j}\left[m_{v} w_{j t n}+\sum_{i} q_{i j} y_{i j t n}\right]\right)+\sum_{j} \sum_{l} c_{l} o_{j l}^{1}+c_{v} v^{1}\right] \\
\text { s.t. } \sum_{l} o_{j l}^{1} \leq 1, j \in J \\
\sum_{l}\left(o_{j l s n}^{2}+u_{j l s n}\right) \leq 1, j \in J, s \in S_{2}, n \in N
\end{gathered}
$$

The objective function is the expected discounted total cost, which consists of the capital cost, operation and maintenance cost, and assignment cost. Inequalities in Eq. (20) and (21) indicate that phasing deployment and capacity expansion cannot be exercised at the same site at the same time. That is, only one type of strategic decision regarding the same candidate site will be exercised.

$$
\begin{gathered}
\sum_{l} x_{j l s n}=\sum_{l} U_{l} o_{j l}^{1}, j \in J, s \in S_{1}, n \in N ; \\
\sum_{l} x_{j l s n}=\sum_{l} x_{j l, s-1, n}+\sum_{l} U_{l}\left(o_{j l s n}^{2}+u_{j l s n}\right), j \in J, s \in S_{2}, n \in N ;
\end{gathered}
$$

Equations in Eq. (22) and (23) represent the numerical relationship between auxiliary variables and initial configurations, as well as between auxiliary variables. Specifically, they reveal the changes in capacity at each site over the life cycle.

$$
\begin{gathered}
x_{j, l+1, s n} \leq x_{j l s n}, j \in J, l \in L_{3}, s \in S, n \in N \\
\sum_{l} u_{j l s n} \leq x_{j l, s-1, n}, j \in J, l \in L_{1}, s \in S_{2}, n \in N
\end{gathered}
$$

Inequalities in Eq. (24) and (25) are logical constraints that make the model realistic and practical. That is, if a station has at least phase $l$ capacity, it certainly has phase $l-1$ capacity. The inequality in Eq. (25) shows that expansion cannot be exercised if there is no available station at the target site.

$$
\begin{gathered}
v^{1}=\sum_{j} \sum_{l} x_{j l s n}, s \in S_{1}, n \in N ; \\
v_{s n}^{2}=\sum_{j} \sum_{l} U_{l}\left(o_{j l s n}^{2}+u_{j l s n}\right), s \in S_{2}, n \in N ;
\end{gathered}
$$

Eq. (26) and (27) represents the decision-making dynamics regarding purchasing vehicles. The number of vehicles purchased every time will be equal to the capacity newly deployed.

$$
\sum_{j \in N_{i}} y_{i j t n} \leq 1, i \in I, t \in T, n \in N
$$




$$
\begin{gathered}
y_{i j t n} \leq \sum_{l} x_{j l s n}, i \in I, j \in J, t \in T_{s}, s \in S, n \in N ; \\
y_{i j t n}=y_{i j t m}, i \in I, j \in J, t \in T_{1}, n, m \in N ;
\end{gathered}
$$

Eq. (28)-(30) are the constraints associated with vehicle assignment. In principle, an incident call at district $i$ can only be responded by vehicles allocated to sites neighboring this district. It is further assumed that one district is covered by one and only one station. The last equation is a nonanticipative constraint, implying that vehicle assignment should be the same for all scenarios in tactical period one.

$$
\begin{gathered}
\sum_{i \in M_{j}} d_{i t n} y_{i j t n}-\sum_{l} x_{j l s n}=\omega_{j t n}-\xi_{j t n}, j \in J, t \in T_{s}, s \in S, n \in N ; \\
\sum_{t} \sum_{j} \sum_{l}\left(x_{j l s n}-\xi_{j t n}\right) \geq \operatorname{CoV} \sum_{t} \sum_{i} d_{i t n}, n \in N ;
\end{gathered}
$$

The inequality on the left-hand side in Eq. (32) denotes the effective capacity of the entire system, which does not necessarily equal the total capacity deployed up to now, due to the assumption of vehicle assignment. Auxiliary variable $\omega_{j t n}$ is greater than zero if the capacity deployed at site $j$ is less than the number of incidents, while $\xi_{j t n}$ has the opposite meaning.

$$
\begin{gathered}
h \sum_{t \in T_{s}} \omega_{j t n}-\delta_{j}^{d} \leq M \sum_{l} u_{j l s n}-\varepsilon+M x_{j k, s-1, n}, j \in J, k \in L_{2}, s \in S_{2}, n \in N \\
h \sum_{t \in T_{s}} \omega_{j t n}-\delta_{j}^{d} \geq M\left(\sum_{l} u_{j l s n}-1\right)-M x_{j k, s-1, n}, j \in J, k \in L_{2}, s \in S_{2}, n \in N \\
\sum_{t \in T_{s}} d_{i t n} \leq \delta_{j}^{o}-\varepsilon+M \sum_{l} o_{j l s n}^{2}+M x_{j l, s-1, n}, i=j, j \in J, k \in L_{1}, s \in S_{2}, n \in N \\
\sum_{t \in T_{s}} d_{i t n} \geq \delta_{j}^{o}-M\left(\sum_{l} o_{j l s n}^{2}-1\right)-M x_{j l, s-1, n}, i=j, j \in J, k \in L_{1}, s \in S_{2}, n \in N
\end{gathered}
$$

Eq. (33)-(36) are constraints regarding flexibility strategies of capacity expansion and phasing deployment. It should be noted that flexibility will be exercised if and only if the conditions are satisfied - including the decision rules (i.e., whether or not the threshold is reached) and real situations (i.e., whether or not the site is empty). This is a relaxed form of non-anticipative constraint. Even if the incident rates are not exactly the same in two scenarios, the flexibility may still be exercised in both scenarios as long as they satisfy the condition (i.e. both rates are greater than the threshold).

$$
\sum_{l} o_{j l s n}^{2} \leq U_{l} \sum_{l} o_{j l s n}^{2} \leq|L| \sum_{l} o_{j l s n}^{2}, j \in J, s \in S_{2}, n \in N
$$




$$
\begin{gathered}
1-\sum_{l} o_{j l s n}^{2} \leq o_{j}^{o}-U_{l} \sum_{l} o_{j l s n}^{2} \leq|L|\left(1-\sum_{l} o_{j l s n}^{2}\right), j \in J, s \in S_{2}, n \in N ; \\
\sum_{l} u_{j l s n} \leq U_{l} \sum_{l} u_{j l s n} \leq|L| \sum_{l} u_{j l s n}, j \in J, s \in S_{2}, n \in N ; \\
1-\sum_{l} u_{j l s n} \leq o_{j}^{u}-U_{l} \sum_{l} u_{j l s n} \leq|L|\left(1-\sum_{l} u_{j l s n}\right), j \in J, s \in S_{2}, n \in N ;
\end{gathered}
$$

The last Eq. (37)-(40) ensures that the capacity deployed in both strategies are consistent at each site throughout the whole study life. That is, the capacity deployed at site $j$ at strategic period $t_{1}$ due to phasing deployment should be the same as the capacity deployed at this site at any other strategic period $t$ where $t \neq t_{1}$. Instead of deploying different capacity at each possible deployment, the model suggests system operators to deploy the same capacity over time if the flexibility is exercised. This is to reduce the complexity of the output and make the solution easier to implement in practice. 
Appendix 3. Table of assumptions

Table 7. List of assumptions of input parameters for the numerical analysis

\begin{tabular}{|c|c|c|}
\hline Parameters & Values & Definitions \\
\hline$S$ & 10 & Number of strategic periods in the life cycle \\
\hline$T$ & 40 & Number of tactical periods in the life cycle \\
\hline$E_{S}$ & 1 year & The length of a strategic period \\
\hline$E_{T}$ & 3 months & The length of a tactical period \\
\hline$N$ & 10 & Number of scenarios in the optimization model \\
\hline$L$ & 4 & Maximal phase of an emergency station \\
\hline$U_{l}$ & $1,2,3,4$ & Unit capacity of a phase $l$ station \\
\hline$c_{l}$ & $\begin{array}{l}\$ 2.00, \\
\$ 6.96^{1}\end{array} \quad \$ 3.73, \quad \$ 5.38$ & Cost associated with installing a phase $l$ station \\
\hline$m_{l}$ & $\begin{array}{l}\$ 0.01, \quad \$ 0.02, \quad \$ 0.03 \\
\$ 0.04^{1}\end{array}$ & $\begin{array}{l}\text { Operations and maintenance cost of a phase } l \\
\text { station per tactical period }\end{array}$ \\
\hline$c_{u}$ & $\$ 2.00, \$ 3.73, \$ 5.38^{1}$ & Cost associated with capacity expansion \\
\hline$c_{v}$ & $\$ 0.1^{1}$ & $\begin{array}{l}\text { Cost associated with purchasing an emergency } \\
\text { vehicle }\end{array}$ \\
\hline$m_{v}$ & $\$ 0.01^{1}$ & Maintenance cost of a vehicle per tactical period \\
\hline$r_{S}$ & $12 \%$ & Discount rate per strategic period \\
\hline$r_{t}$ & $2.83 \%$ & Discount rate per tactical period \\
\hline$h$ & 2160 & Number of hours per tactical period (i.e., $90 \times 24$ ) \\
\hline$\varepsilon, M$ & $10^{-3}, 10^{6}$ & A small tolerance and a very large integer \\
\hline$R$ & 10 units & Coverage radius \\
\hline$t_{u}$ & 4 & $\begin{array}{l}\text { Number of tactical periods for observation } \\
\text { regarding capacity expansion }\end{array}$ \\
\hline$t_{o}$ & 4 & $\begin{array}{l}\text { Number of tactical periods for observation } \\
\text { regarding phasing deployment }\end{array}$ \\
\hline
\end{tabular}

${ }^{1}$ : \$million US dollars. 